\title{
Experimental Investigation of the Pressure, Force, and Torque Characteristics of a Rigid Flapping Wing
}

\author{
Derrick Yeo* ${ }^{1}$, Ella M. Atkins ${ }^{*^{2}}$, Luis P. Bernal ${ }^{* 3}$ and Wei Shyy $*^{\dagger 4}$ \\ *University of Michigan, Ann Arbor, Michigan, 48109, U.S.A \\ $\dagger$ Hong Kong University of Science and Technology, Kowloon, Hong Kong
}

Pressure measurements over flapping wings are less commonly available due to the difficulties associated with instrumenting them. This represents an important limitation of the aerodynamic data available for the development of self-contained flapping wing vehicle autopilots and for researchers working on the aerodynamics of bio-inspired flapping wings. This paper describes the design, construction, and testing of a customized flap stand, and a distributed pressure sensing system embedded in a set of rigid flapping wings to provide high speed 'on-board' flow measurements. While the hovering condition is the main focus, the setup is applicable to forward flight conditions as well. Data processing techniques are described along with the method used to measure pressure distributions over the wings. A series of flap tests were conducted in still air, with pressures measured only in air and force and torque measurements taken both in air and in a vacuum. Comparisons between the pressure-based estimates of aerodynamic loads and direct measurements taken by the force instrumentation are presented. The comparisons suggest the pressure-based system provides reasonable estimates of aerodynamic loads but is limited by the frequency response of the pressure lines used.

\section{Nomenclature}

$\begin{array}{ll}\mathrm{b} & =\text { wingspan, } \mathrm{m} \\ c_{m} & =\text { mean chord, } \mathrm{m} \\ h & =\text { stroke amplitude, } \mathrm{m} \\ f & =\text { frequency of oscillation, } \mathrm{Hz} \\ \operatorname{Re} & =\text { Reynolds number, } \operatorname{Re}=\frac{V_{t i p} \cdot c_{m}}{v} \\ k & =\text { Reduced Frequency, } k=\frac{f \cdot \pi \cdot c_{m}}{V_{t i p}} \\ \beta & =\text { wing flapping angle } \\ v & =\text { kinematic viscosity, } \mathrm{m}^{2} / \mathrm{s}\end{array}$

\section{Introduction}

$\mathrm{T}$ he decreasing sizes of modern unmanned aircraft systems (UAS) challenge the abilities of traditional fixed wing designs to perform adequately. As the size of a conventional fixed wing flyer decreases, low Reynolds number effects limit its ability to generate lift, requiring higher airspeeds that might not be practical for many UAS

\footnotetext{
${ }^{1} \mathrm{PhD}$ student, Aerospace Engineering Dept, University of Michigan, Ann Arbor, MI

${ }^{2}$ Associate Professor, Aerospace Engineering Dept, University of Michigan, Ann Arbor, MI, Associate Fellow.

${ }^{3}$ Associate Professor, Aerospace Engineering Dept, University of Michigan, Ann Arbor, MI

${ }^{3}$ Provost \& Chair Professor, Department of Mechanical Engineering, Hong Kong University of Science and Technology, Hong Kong; also Adjunct Professor, Department of Aerospace Engineering, University of Michigan; AIAA Fellow.
} 
applications. Flapping wing systems, as evolved by nature, have been observed to enjoy aerodynamic benefits instead of penalties at these smaller scales [1] allowing small UAS to operate at lower airspeeds. These potential benefits are accompanied by considerable challenges due to the unsteady aerodynamics associated with flapping flight. This paper documents ongoing work in an experimental investigation to assess the viability of using pressure based aerodynamic sensing for real-time estimation of the aerodynamic forces acting on a flapping wing vehicle. It presents an experimental investigation of the forces and torques generated by a set of instrumented flapping wings featuring pressure based measurements. The instrumented wings are designed to be rigid in order to facilitate the isolation of aerodynamic loads from inertial loads measured in vacuum.

Flapping wing vehicles have been built and tested by the engineering community, from micro $[2,3]$ to small scale $\sim 10 \mathrm{~cm}$ vehicles $[4,5,6]$, to tests done on larger scale vehicles[7, 8,9] with wingspans $\sim 1 \mathrm{~m}$. Autonomous ornithopter scale flapping wing vehicle flight has been successfully conducted by researchers utilizing extended fixed wing autopilot formulations. Kranashita[10] incorporated a simple Paparrazi autopilot unit on a Cybird P2 ornithopter and demonstrated autonomous, way point tracking flight. More recently, Lee et al [9] designed and built a flapping wing platform, SF-2 which flew autonomously with a more complete inertial measurement sensor suite.

Most current flapping wing vehicles rely on moving surfaces adapted from traditional aircraft designs for attitude control. A single tail surface typically provides pitch and directional control and the main wings are not actuated beyond their fixed flapping stroke [11]. While such designs have successfully, both for recreational and research applications, $[4,5,8,9,10]$ they fail to afford ornithopters the agility of fixed or rotary wing vehicles. Even the Festo Seagull [12] which utilizes the most advanced wing actuation to date cannot mimic the full capabilities of its biological namesake. Incorporating attitude control actuation on the moving wings of an ornithopter presents even more challenges due to constantly changing forces and inherently non-linear aerodynamics, but offer the possibility of improved aerobatic performance.

In order to simulate the aerodynamics of flapping wing flight, researchers have used full Navier-Stokes Computational Fluid Dynamics (CFD) solvers and those of potential flow, discrete vortex type methods [13,14]. The thin membrane wings used by successful flapping wing flyers and the kinematics of the flapping process induces considerable coupling between fluid and structural dynamics during flapping, thus it is difficult to study one aspect without considering the other. Aerodynamic models must be coupled with structural solvers in order to describe the physics of a practical flapping wing. Researchers have coupled both CFD and discrete vortex methods with structural solvers $[15,16]$ that generate results that could be validated against existing experimental data. These computational frameworks also enable the development of new scaling relationships that provide estimates of expected forces from the known parameters of a flapping wing [17]. While these analyses include the additional complexities of flexibility and go beyond the current scope of the rigid wing work presented in this paper, results from these studies will have significant implications on future implementations of similar pressure based measurement systems that require significant changes to the mass and flexibility properties of a practical flexible wing.

In terms of gathering aerodynamic data, current experimental work has ranged from measurements of live biological flyers from [18,19], water tunnel based studies of characteristic fluid flow fields $[20,21]$ to tethered flight testing of existing flight vehicles of sizes ranging from insect-like scales [3,4] to those of bird-type scales [22]. One challenge in measuring aerodynamic forces is separation of aerodynamic forces from the inertial forces due to the moving wing structure. In order to do this, the inertial forces and torques due only to the wings' motion must be measured. Two possible approaches include the use of mass-tuned 'inertial only' wings that have a negligible surface area, and running tests in a vacuum environment. Massey et al [22] built 'inertial wings' from aluminiun pipes and used those as a structural load calibration to back out aerodynamic forces. Singh and $\mathrm{Wu}[23,24]$ ran tests in a vacuum environment to obtain inertial loads. In these studies, measurements of the flow field are typically achieved using PIV instrumentation as in $[20,21]$. Researchers $[25,26]$ have also taken pressure measurements across the surface of a flapping wing, providing experimental data that can potentially be accessible to an onboard autopilot.

In developing an early controller, Deng [27,28] extended the standard aircraft type model and time-averaged forces and implemented a switching controller of an insect type flyer near hover. Bolender [29] treated flapping wing micro air vehicles (FWMAV) as a collection of four rigid bodies: wings (2), body and tail, and derived the corresponding equations of motion using Kane's equations. Researchers have used both experimental and 
computational results to formulate control strategies that aim to recreate a degree of the maneuverability of biological fliers. Doman [30] used aerodynamic coefficients from oil tank experimental data to investigate a splitcycle wing beat type control with a non-linear dynamics model [32] while Deng [28] used CFD results in deriving the equations of motion of a FWMAV. Having access to real-time aerodynamic data can be beneficial in developing control algorithms for autopilots in situations where unsteady aerodynamic forces are difficult to predict, like during aggressive maneuvers. In previous work [25] the authors have demonstrated improvements to a simple fixed wing transition-to-hover autopilot by adding the ability to recognize stall during significant changes in aerodynamic state. In flapping wing flight, the forces acting on the vehicle are always changing and flow separation is a constant flow feature, representing an even greater challenge for inertial based autopilot formulations. Kranashita in [12] suggested that a lack of accurate aerodynamic models for instant aerodynamic forces on flapping wings remains the main obstacle for a formal mathematical analysis of flight dynamics and control of these vehicles.

This research describes recent progress in implementing a pressure based aerodynamic data system for flapping wing vehicles. This paper describes a series of flap tests in both air and vacuum that have been run in order to measure the aerodynamic loads generated by a set of rigid elliptical wings using both a force torque sensor and the aerodynamic data system. Aerodynamic measurements, specifically an array of pressure sensors across the flapping surfaces, provide real-time pressure measurements. While such measurements cannot provide a complete picture of the flow field like PIV, they can be acquired and processed in real time and do not require an external test setup. Since all the necessary flow instrumentation can be carried onboard, this makes the pressure based approach to flow measurements inherently more feasible for use in an autopilot. Below the set of instrumented flapping wings used for this work are described. The experimental process is then outlined, including tests in a wind tunnel and in a vacuum chamber using ornithopter type flapping mechanics. The data analysis techniques used are then described. Finally, comparisons between experimental and computational results are made, followed by comments on the viability of a pressure based aerodynamic data system for flapping wing vehicles.

Pressure measurements have been used before in this context. In purely lab based experimental work, Hilaire and Carta[32] have taken pressure measurements over a range of oscillating wings with a symmetrical airfoil with different planforms. Green and Smits [33] have studied pressure distribution over a 3D , 2 degree of freedom foil oscillating in a water stream and formulated a new propulsion scaling based on pressure. On UAS platforms, the authors [25] have implemented a pressure based aerodynamic sensing system and integrated its data into a levelflight to hover transition guidance law. In a collaborative effort, the most recent AFOSR MURI group [26] have developed micro-tuft type mems sensors for flow direction and magnitude detection on a fixed-wing UAS. Most recently, researchers from the University of Tokyo [34] have demonstrated the near term potential of this sensing strategy by flight testing an insect scale flyer with integrated pressure sensors in its flexible wing membranes. The distinction of our pressure measurements is in the number of measurements and its correlation with forces and torques to facilitate model validation. In our previous work, a platform for the wind tunnel testing of flapping wing vehicles that enables high speed pressure sensing over the wings and force-torque measurements was developed by the authors [35]. Preliminary pressure data was presented that served as a proof of concept for pressure based aerodynamic sensing. Two sets of test wings were instrumented by installing pressure ports at locations that served to discretize the wings surface and provide an experimental 'pressure map'. Pressure histories over averaged flap cycles corresponded well to predictions made using simple panel method codes for three different test cases. In addressing issues with flap stroke inconsistencies, the authors developed a new robust flapping wing platform and used the VICON motion capture system to verify its ability to provide dependable and repeatable kinematics [36].

The objective for the present second-generation series of tests is to obtain high quality force torque measurements of the aerodynamic forces. In the case of a rigid wing, this is challenging as aerodynamic forces generally represent a small portion of the total loads. To separate inertial loads from aerodynamic forces, experiments in vacuum were conducted. High quality wing position data have also enabled Force-Torque and Pressure test runs to be performed separately, addressing the possible problem of pressure lines affecting force readings. A frequency domain analysis is used to gain insight on filtering techniques for experimental data. While the setup is intended for forward flight, the present experiments focus on hover in order to develop data processing methods before introducing a free stream. Some key physical parameters of the flapping wing tests conducted are summarized in Table 1. 
Table 1: Key Physical Dimensions of Flapping Wing Test Cases

\begin{tabular}{|c|c|c|}
\hline Physical Length & Symbol & Value $(\mathrm{mm})$ \\
\hline Half Span & $\mathrm{b}$ & 213 \\
Root Chord & $\mathrm{C}$ & 72 \\
Mean Chord & $\mathrm{C}_{\mathrm{m}}$ & 61 \\
Plunge Amplitude & $\mathrm{h}$ & 157 \\
\hline
\end{tabular}

Based on these physical characteristics, the area and mean chord of the wing is computed in the traditional manner for an elliptical planform but the half span is considered when calculating the aspect ratio in this study. In the absence of a free stream, the tip velocity of the flapping wing is used as the reference velocity when considering the dimensionless numbers. In this paper, the average tip speeds are considered. At hover, the Reynolds number that compares of the ratio of inertial forces to viscous forces for the flow phenomena in question is defined as shown in Equation (1) and depends on the flapping frequency through $\mathrm{V}_{\text {tip }}$.

$$
\operatorname{Re}=\frac{V_{t i p} \cdot c_{m}}{v}
$$

When considering unsteady flow phenomena, the reduced frequency offers an indication of how unsteady the flow field around the object is. When considering a flapping wing in forward flight, it can be thought of as a comparison between how quickly flow disturbances are convected by the free stream and the speed of the motion causing the disturbances. However at hover with no free stream, the mean half stroke tip speed of the wing is used as the reference velocity instead, as shown in Equation (2). In this situation, the reduced frequency can be thought of more as a comparison between the typical length scale of the flow disturbance and the characteristic length of the object causing it. Since this definition relates tip speed to the mean chord, only the geometry of the wing and stroke kinematics influence the reduced frequency at hover.

$$
k=\frac{f \cdot \pi \cdot c_{m}}{V_{t i p}}
$$

The non-dimensional parameters that represent the flapping tests conducted are presented in Table 2 . In the following sections, we summarize the current mechanical design, sensor hardware, experimental strategy, data processing methods and present a brief description of experimental results.

Table 2: Key Dimensionless Parameters of Current Experimental Tes
\begin{tabular}{|c|c|c|}
\hline Dimensionless Parameter & Symbol & Value \\
\hline & & \\
Aspect Ratio & AR & 3.75 \\
Thickness Ratio & $\mathrm{h}^{*}$ & 0.05 \\
Reynolds Number & $R e$ & $3.5-5.5 \times 10^{3}$ \\
Reduced Frequency & $k$ & 0.62 \\
\hline
\end{tabular}

\section{Flapstand Sensor Configuration and Mechanical Design}

A custom flap stand has been previously developed by the authors to take synchronized, high speed pressure, FT and wing location readings during a wind tunnel test [35]. Primary instrumentation includes a bank of low pressure MEMS pressure sensors and a six-axis force-torque (FT) sensor. An overall schematic of the flap stand is shown in the figure below. The test model, FT sensor, and a pitot probe are supported by an adjustable main arm to accommodate different wind tunnel test sections for different tests. During wind tunnel tests, pressure lines and electrical wiring are routed to two aerodynamic shells located behind the stand which house the embedded computer, data acquisition hardware and pressure sensors. During vacuum chamber tests, the flap stand is mounted 
inside the chamber and electrical feedthroughs connect the test stand to the embedded computer outside. FT readings are taken using an ATI nano17 six-axis FT sensor to which the flapping mechanics are mounted. The sensor tip is connected to an ATI IFPS-1 interface/power supply unit and the voltages reported by the interface board are read by the $\mathrm{AD}$ converter on the embedded computer. Data acquisition is run at $1 \mathrm{kHz}$ and the embedded computer provides 16bit resolution on 32channels.

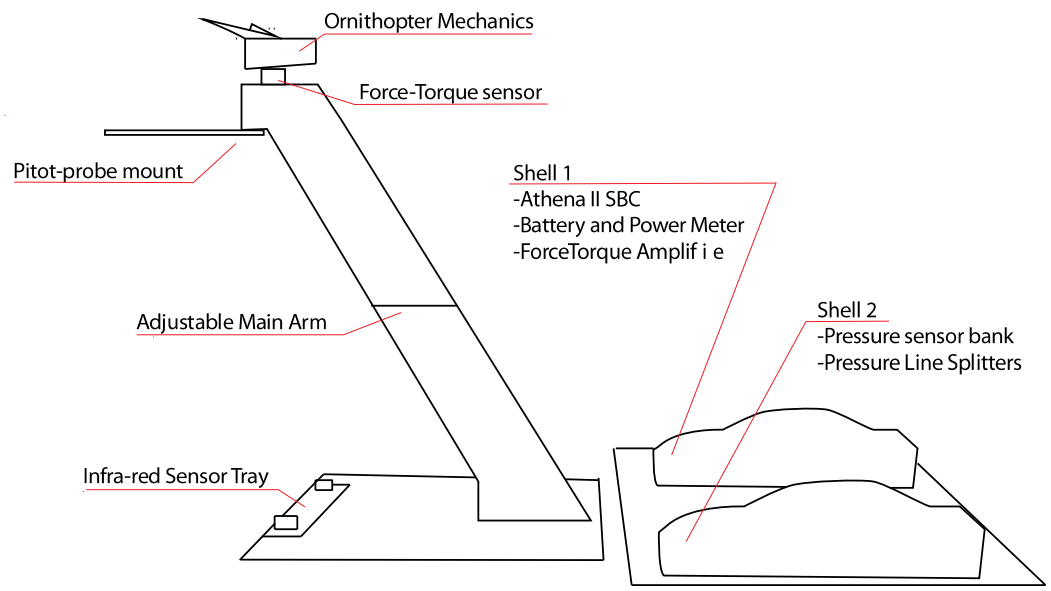

Figure 1: Wind Tunnel Test Setup Schematic [35]

A custom set of flap mechanics were designed for this work as previous experience with commercially manufactured Cybird mechanics showed that reliability and stroke repeatability would not be compatible with a successful multi-phase test plan as the wing stroke would change between tests. The set of mechanics designed for the test stand were built to provide a reliable and repeatable flap stroke. The resulting flapping stroke replicates those found on typical hobby ornithopters and can be finely adjusted for amplitude and flap angle through the final linkage geometry. It is constructed from stacked fiberglass sheets and uses high precision bearings on all rotating parts to prevent mechanical slop from causing inconsistencies in the resulting stroke. The two-stage transmission was designed to run off existing RC hobby drive hardware which are relatively easy to source. The spur gear of the first reduction stage was chosen to mesh with Radio Control hobby helicopter 48-pitch pinion gears common for applications of this size, making the mechanics compatible with a wide selection of small RC helicopter motors and hobby motor controllers. For these tests, a generic '300' size 3800KV brushless motor and a 40amp Hobby Wing motor controller were used along with a two cell 4000mah lithium polymer battery for power. A six tooth 32-pitch pinion rod was interfaced with this spur gear and used to drive the final crank assembly.

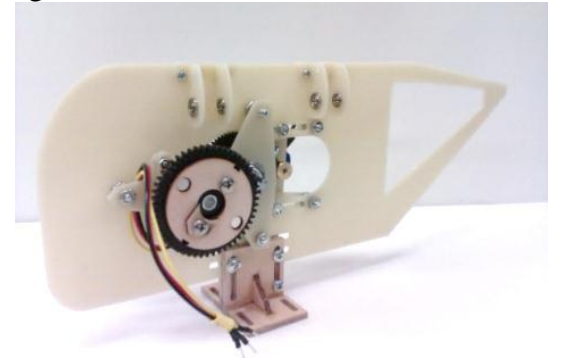

Figure 2. Flapping Transmission with Integrated Encoder System[36]

The final crank also accommodates a pair of magnets for wing position feedback. A hall-effect sensor detects the passing of the magnet and provides two position updates for every revolution of the final crank. The encoder signal is split between the embedded computer for data acquisition, and a second microcontroller based flap governor that controls the flapping frequency of the wings. The custom built and microcontroller board uses the wind encoder signal to run a closed loop, integrating feedback controller to generate appropriate commands to the RC motor controller. The board takes command signals from an infra-red television remote control and displays a real time update of crank status, current flap frequency and desired reference frequency on an attached LCD display. The firmware also includes a controller-hold mode of operation where the feedback controller is turned off. 


\section{Wing Instrumentation and Kinematics}

For this study, a flat plate Zimmerman planform was chosen. The wings were built by laminating sheets of thin balsa wood. Pressure readings are taken through ports located on the surface of the flapping wings. The pressure ports correspond to a coarse discretization of the wing surface as shown in the diagram below. Measurements at each port provide the differential pressure across the top and bottom surface of the wing at that point and can be simply multiplied by the area around its corresponding wing area and integrated over the wing to provide an estimate of the aerodynamic forces being generated through the flap stroke.

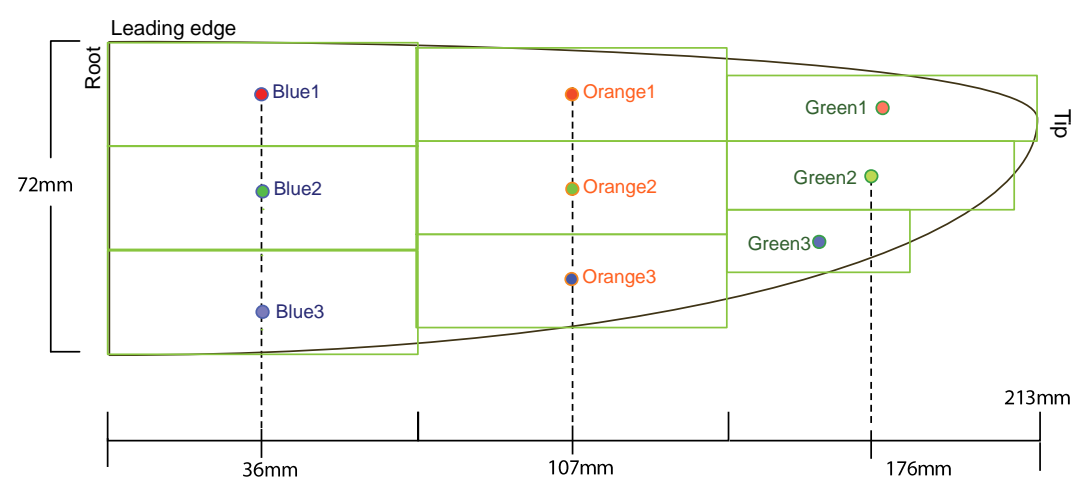

Figure 3: Wing Overview and Pressure Port Locations

Using a technique previously developed by the authors [35] small pressure lines have been embedded into the laminated balsa sheet wings. Due to the thickness of the lines, the wings have a planar cross section that has a thickness of $4 \%$ of the root chord. As constructed, the wings make up a full span of $426 \mathrm{~mm}$ and an aspect ratio of 7.5. When mounted on the flapping mechanics, these wings have a plunge amplitude $h$ of $157 \mathrm{~mm}$. The pressure ports are connected to a bank of pressure sensors located on the flap stand. The sensors used are $0.25 \mathrm{Inch}-\mathrm{D} 4 \mathrm{~V}$ differential sensors manufactured by All Sensors Corporation. These sensors are pre-amplified and provide a linear output voltage across its range. The individual sensors used were re-calibrated in the University of Michigan undergraduate $2 \times 2$ wind tunnel lab with an inclined manometer.

In order for the multi-phase experimental approach to be successful, the wing kinematics must be repeatable and predictable throughout the complete set of tests. If not, the loads and pressures measured at different phases of the testing cannot be reliably related to each other. The wings used for this study are rigid which allows aero-elastic effects to be neglected for the purpose of tracking wing deflections and computing aerodynamic loads. With no aero-elastic coupling, the wing deflections in both air and vacuum will be identical allowing the inertial loads measured in the vacuum to accurately represent those encountered in air. Rigid wings also simplify the process of estimating resultant aerodynamic forces from pressure measurements as the orientation of the wing surface is always known. The wing kinematics were simulated using a 3D linkage code for the mechanics and assumed the wings were rigid. As the wings were built out of balsa wood, their rigidity at the flapping frequencies needed to be tested.

A reference set of wings were instrumented with reflective targets and using the VICON motion capture system, position histories of various locations on the wing surface were measured at different frequencies in hover. The displacement histories were examined for un-predictable and unwanted wing deformations as the loads varied with flap frequency [36]. It was that no inconsistencies in wing deflection were apparent between different frequencies and that the linkage output correctly predicted the flapping angle of the wing during its stroke. A comparison between experimental and numerical predictions is shown below. The data collected showed that the rigid wing assumption was sound and that the designed flap mechanics were repeatable and reliable. 

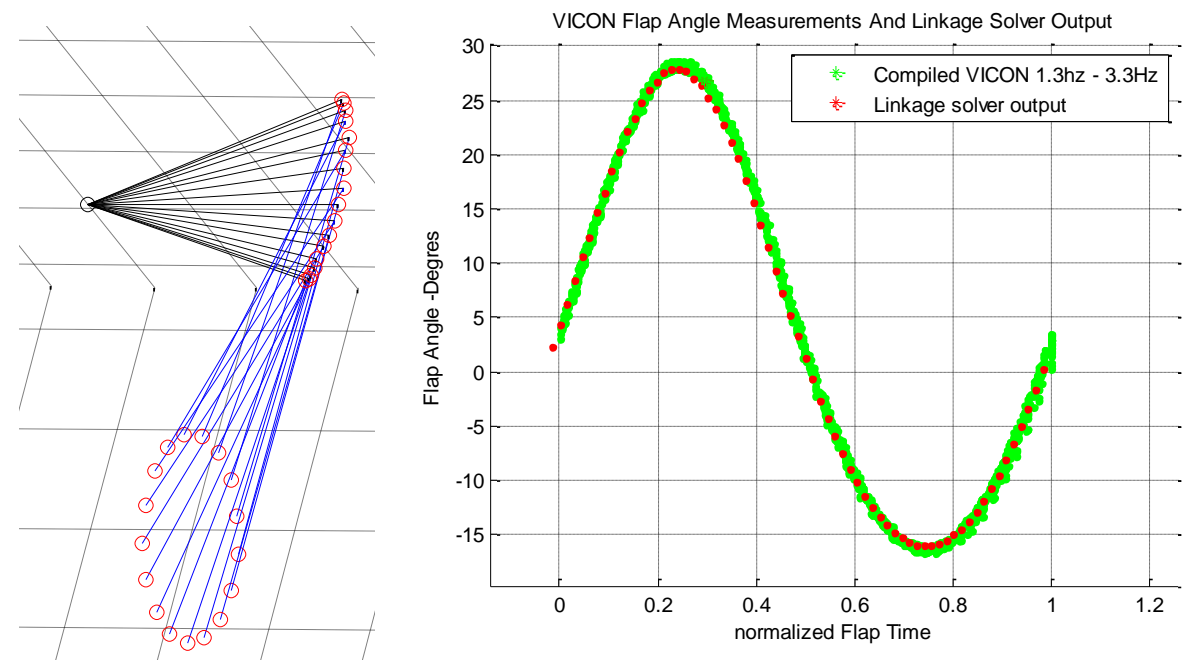

Figure 4: Sample Linkage Solver Output with 17 Crank Steps for Clarity. 3D View Showing Crank Positions with Red Dots and Wing Positions with Black Lines (Left). Corresponding Computed Wing Flap Angle $\beta$ Output at Given Crank Positions (Right) and comparison to VICON data. [36]

The flap stroke resulting from the 4-bar crank mechanism is asymmetrical and is easily represented by a $4^{\text {th }}$ order Fourier fit. This process is described in more detail in our previous work [36] but a summary of the relevant coefficients used are presented in table 3 and are used as shown in Equation (3) below.

Table 3: Fourier Fit Coefficients of Wing Kinematics

\begin{tabular}{|c|c|c|c|}
\hline Coefficient & Value & Coefficient & Value \\
\hline $\mathrm{a} 0$ & 0.0354 & a3 & $-8.90 \mathrm{E}-07$ \\
\hline a1 & 4.10E-05 & b3 & -0.0035 \\
\hline b1 & 0.3793 & a4 & 0.00046 \\
\hline a2 & -0.0322 & b4 & $-3.60 \mathrm{E}-06$ \\
\hline b2 & $-1.95 \mathrm{E}-06$ & w & 6.231 \\
\hline
\end{tabular}

$$
\beta(t)=a_{0}+a_{1} \cos (f t w)+b_{1} \sin (f t w)+\ldots .+a_{n} \cos (n \cdot f t w)+b_{n} \sin (n \cdot f t w)
$$

With FT measurements, matching the mass balancing between the different wings was important. If the wings mounted on the left and right of the vehicle were unbalanced, significant moments and errors in vertical forces can be expected during a flap stroke. In order to avoid this, a scientific scale and a knife edge were used to match the wings for mass and cg location. First, the all wings were constructed in an identical fashion, minimizing inconsistencies to those due to material and manufacturing differences. To overcome these slight differences, measured lengths of copper wire were inserted down the length of each wing for both weight and CG balance point. The built in pathways for the integrated pressure lines allowed this to be done in a consistent manner. Table 4 summarizes the final masses of the wings used for these measurements.

Table 4: Wing Masses and Left/Right Imbalance

\begin{tabular}{|c|c|c|}
\hline Wing Id & Mass & \multirow{2}{*}{ L/R error } \\
\hline Left Primary & 14.45 & \multirow{2}{*}{$0.60 \%$} \\
\hline Right Primary & 14.35 & \\
\hline L Spare & 15.58 & \\
\hline R Spare & 15.44 & $0.90 \%$ \\
\hline Pressure Wing & $16^{*}$ & (NA) \\
\hline
\end{tabular}


All wings were constructed identically with integrated pressure lines and ports. However, only one set of Pressure Wings included full lengths of external pressure connections extending from them that allow their internal pressure ports to be connected to the pressure sensor bank. These were not used for FT measurements in either the vacuum chamber or in the wind tunnel as lab testing showed that the pressure lines caused unwanted noise and inconsistencies in the force measurements. To avoid vibrations during pressure measurement tests, the pressure wings and the extended pressure lines were balanced approximately with the others using the same technique described above.

\section{Experimental Procedure and Test Plan}

Since our primary interest in this study is to assess the viability of using pressure measurements to estimate the aerodynamic forces on a flapping wing, it is critical that these loads be separated from the loads associated with the motion of the wings. To separate aerodynamic forces from inertial loads, multiple tests using the same wing kinematics had to be performed in both vacuum and in wind tunnel environments. Since the rigid wings used are not subject to aeroelastic deformations and the flap stroke used is well documented, the experiment can be run multiple times in different environments and the data from all the individual tests could be cycle averaged and merged using the signal from the magnetic encoder. This allowed us to split our experiment into three separate test phases which are depicted in the schematic below.
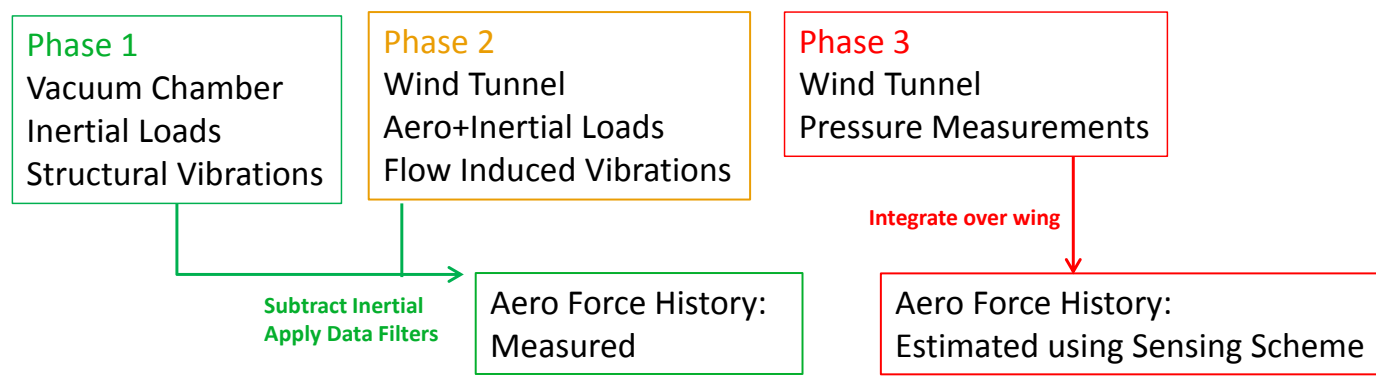

Figure 5: Overview of Revised Experimental Procedure

The first phase involved testing in a vacuum chamber where the forces and torques due only to inertial loads can be measured. The second phase was to be conducted in the wind tunnel with the pressure lines disconnected to enable the full aerodynamic and inertial loads to be measured. The difference between the two readings will be the forces due to aerodynamic loads. The final phase involved taking only pressure measurements and was conducted in the wind tunnel as well but with one of the wings replaced the pressure wing that had the pressure ports connected. By splitting the experiment into these three separate test phases, three sets of data were combined to obtain an ensemble averaged cycle history of inertial loads, aerodynamic loads, wing position and wing pressure histories. The separate phases are described in the following sections.

\section{Vacuum Chamber Measurements of Inertial Loads}

Tests were conducted in the University of Michigan Plasma-dynamics and Electric Propulsion Laboratory (PEPL) 'Junior' vacuum chamber. The pressure was automatically maintained at 8.3 torr which corresponds to $\sim 11 \%$ of atmospheric pressure. Due to the lack of convection cooling available in a vacuum, it was determined that the embedded PC104 computer system had to be left outside the vacuum chamber. It was positioned near the floor of the chamber and connected using electrical feedthroughs and specially built wiring harnesses. Through a series of harness tests, it was determined that the FT sensor, magnetic encoder and new infra-red wing position sensor were not adversely affected by the change in wiring scheme for the vacuum chamber set up. These remained available during vacuum chamber tests.

The micro-controller based flap-governor and remote interface operate at a lower voltage and it was determined they were in no danger of micro-arcing or overheating under a low vacuum. These components remained mounted on the stand when in the vacuum chamber and facilitated flap frequency control. A Sony TV remote control was used to transmit Infra-red command signals through a viewing port and to the microcontrollers on the flag-governor. This 
custom built and programmed infra-red remote control system is convenient in a wind tunnel environment but was critical for vacuum chamber testing. With a limited number of electrical feedthroughs, using an infra-red remote allowed changes in the desired reference flap frequency and operational mode to be made from outside the vacuum chamber without requiring additional wiring. The back-lit LCD screen on the stand also remained in vacuum and provided a real-time display of flap frequency and the status of the closed loop controller which could be read through the viewing port.

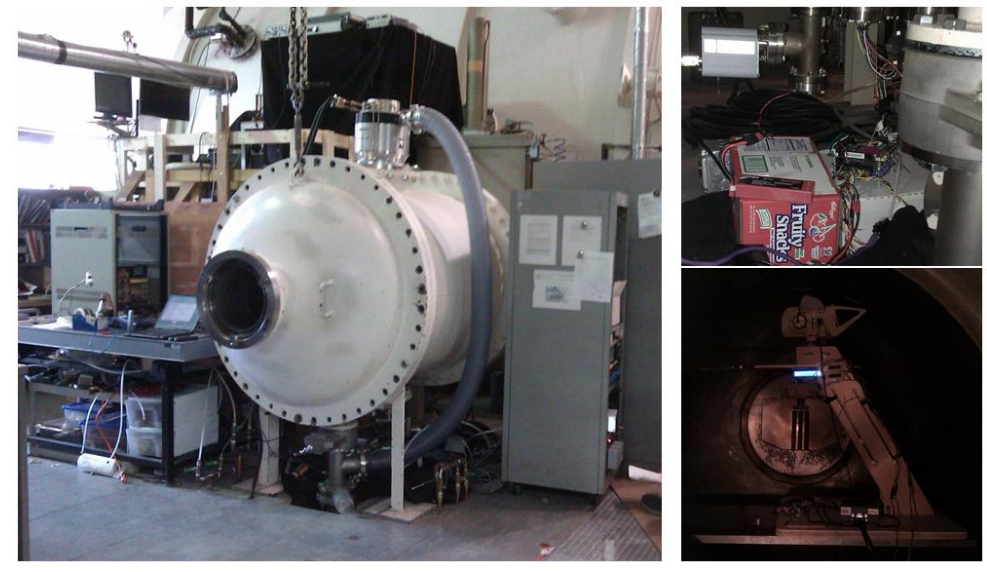

Figure 6: PEPL ‘Junior’ Vacuum Chamber Test setup

One flap test was run immediately after the chamber had been vented to offer a first order check of the results. Due to the tight wall clearances between the vacuum chamber test section and the wing tip path, this set of hover results were not considered to be reliable and was only used as an immediate, first check of the Vacuum results. The plots in figure 7 show averagedvertical force (Fz) histories from the 4 different flap frequencies when in vacuum and at 1 atmosphere. The convention used was for positive vertical forces to be in the direction that created lift in the body frame of the mounted vehicle. These were post-processed using a $6^{\text {th }}$ order Butterworth Low Pass Filter implemented in Matlab with a cut off frequency of double the flap frequency. The selection of these filter settings are discussed in the following data analysis section. While the loads are very similar, there are distinct differences between the vacuum and air measurements at the ends of each half-stroke which showed that the chamber did indeed go into a vacuum and the tests provided a different set of results. With the chamber at 1atm, it was noted that the recorded loads showed slightly increased peak magnitudes and a slight change in phase due to aerodynamic effects. .It can also be noted that due to the asymmetric flap stroke, the resulting inertial loads are not symmetric either with a flatter upward force peak in the second half of the stroke.
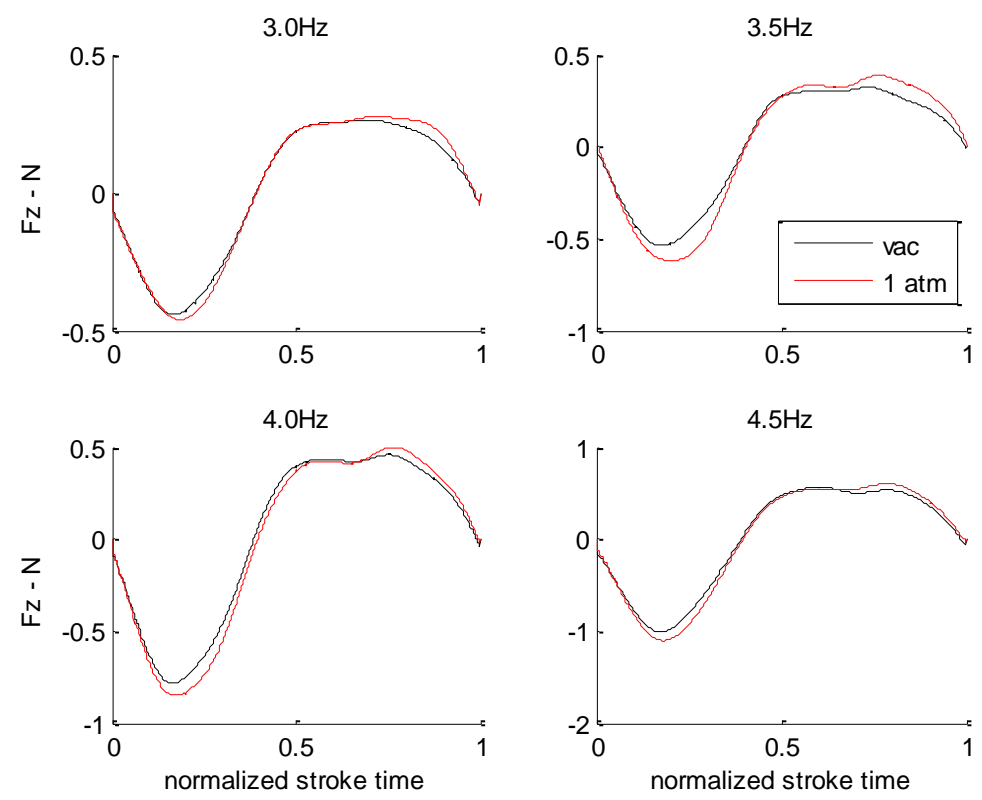

Figure 7: Vacuum Chamber Data Overview 


\section{Wind Tunnel Vertical Force and Pressure Measurements}

Wind tunnel testing was conducted in the UM $5 \mathrm{ft} . \times 7 \mathrm{ft}$. wind tunnel. The flapping stand mounted in the test section is shown in Figure 8. While the aerodynamic data system and overall sensing concept is intended for forward flight, tests were first run at hover in order to develop data processing methods before introducing a free stream. The test stand in the wind tunnel test section is depicted below. In earlier testing, it was noted that the pressure lines exiting the instrumented wing caused significant noise in the force measurements. As such, a second instrumented wing was built solely for the purpose of taking force measurements.

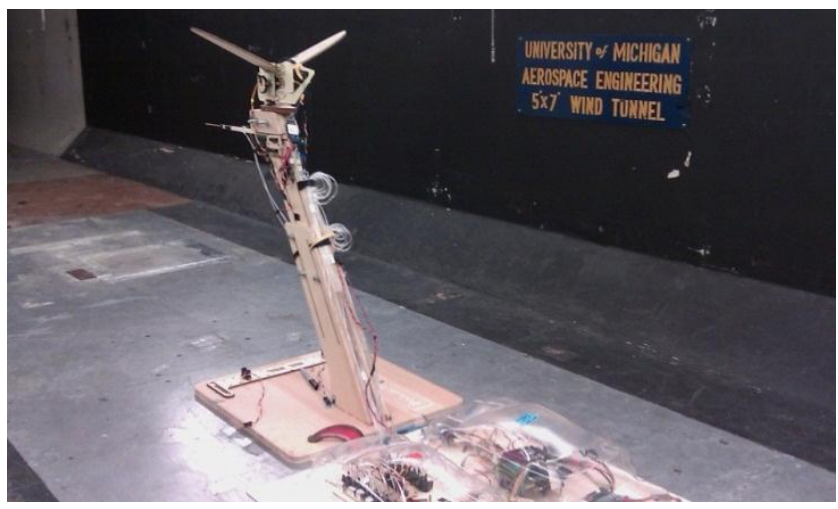

Figure 8: Wind Tunnel Test Setup

Hover data was taken at $3.0 \mathrm{~Hz}, 3.5 \mathrm{~Hz}, 4.0 \mathrm{~Hz}$ and $4.5 \mathrm{~Hz}$. Based on the plunge amplitude of $157 \mathrm{~mm}$ and frequency, these cases correspond to Reynolds numbers between 3500 and 5500 as described earlier. A set of sample results at $4.0 \mathrm{~Hz}$ are shown below where the vertical force measurements are compared against those taken in vacuum on the left. On the right hand side, the pressure port readings throughout the characteristic stroke are shown. The blue, orange and green ports represent pressures from the most inboard, mid-span and most outboard span-wise sections respectively. On each span-wise location, red, green and blue dots denote pressure from the leading edge, mid-chord and trail-edge pressure ports.
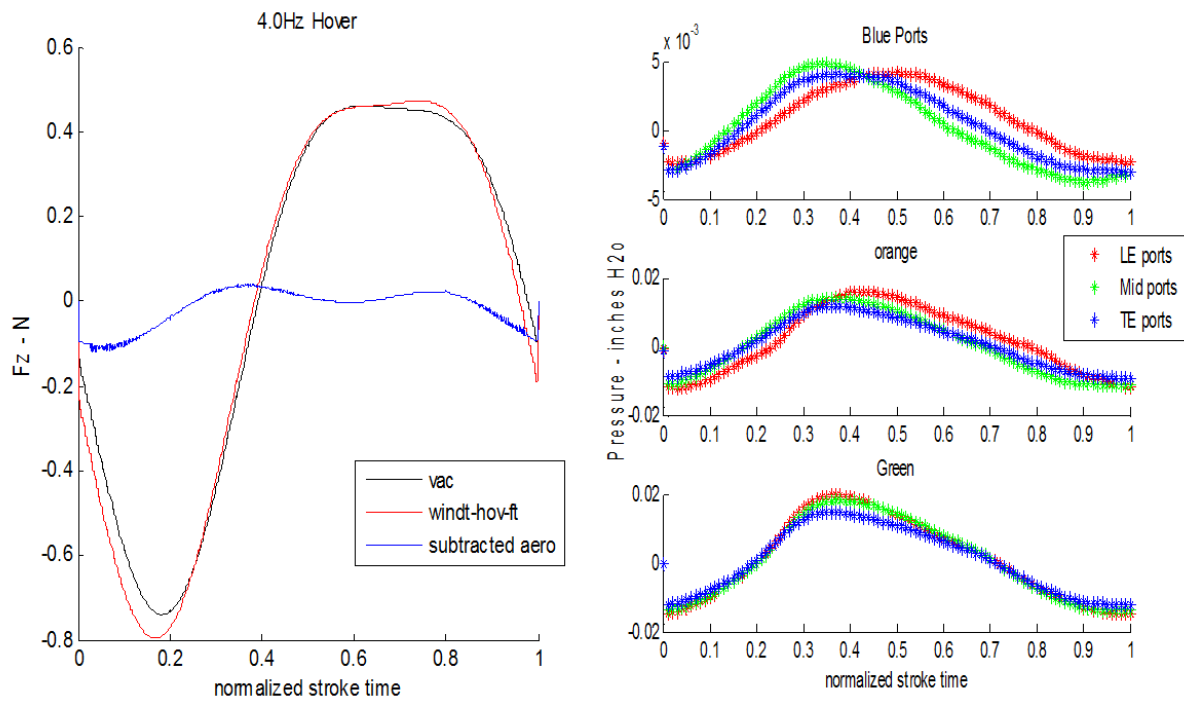

Figure 9: Sample Wind tunnel Force and Pressure measurements - Hover

The force measurements in the vacuum chamber from Phase 1 were used in conjunction to those taken in the wind tunnel FT measurement phase (Phase2) to yield a measurement of the aerodynamic loads. By subtracting the inertial loads from the combined air and inertial loads, a history of the aerodynamic forces of the characteristic stroke are obtained. The measurements from pressure ports in Phase 3 allow the reconstruction of pressure history across the 
wings surface during the test based on the pressure port locations and a coarse discretization of the wing as described in Section III. These pressure measurements are integrated for a second estimate of the aerodynamic loads during a characteristic stroke. The data processing methods used are described in more detail in the following sections.

\section{Pressure Based Aerodynamic Force Measurements}

Pressure data was compiled in a manner described in previous work [36] by the authors. Due to the large volume of relatively consistent raw pressure data, a simple spatial and temporal averaging scheme was used to form the pressure history of a characteristic flap stroke. This technique accounts for slight phase time offsets between individual flap strokes and was amenable to an error characterization. More details on the process used can be found in [36]. A typical set of pressure histories is shown at $\mathrm{f}=3.5 \mathrm{~Hz}$ in the following figure. As mentioned above, the blue, orange and green ports represent pressures from the most inboard, mid-span and most outboard span-wise sections respectively. On each span-wise location, red, green and blue dots denote pressure from the leading edge, mid-chord and trail-edge pressure ports.

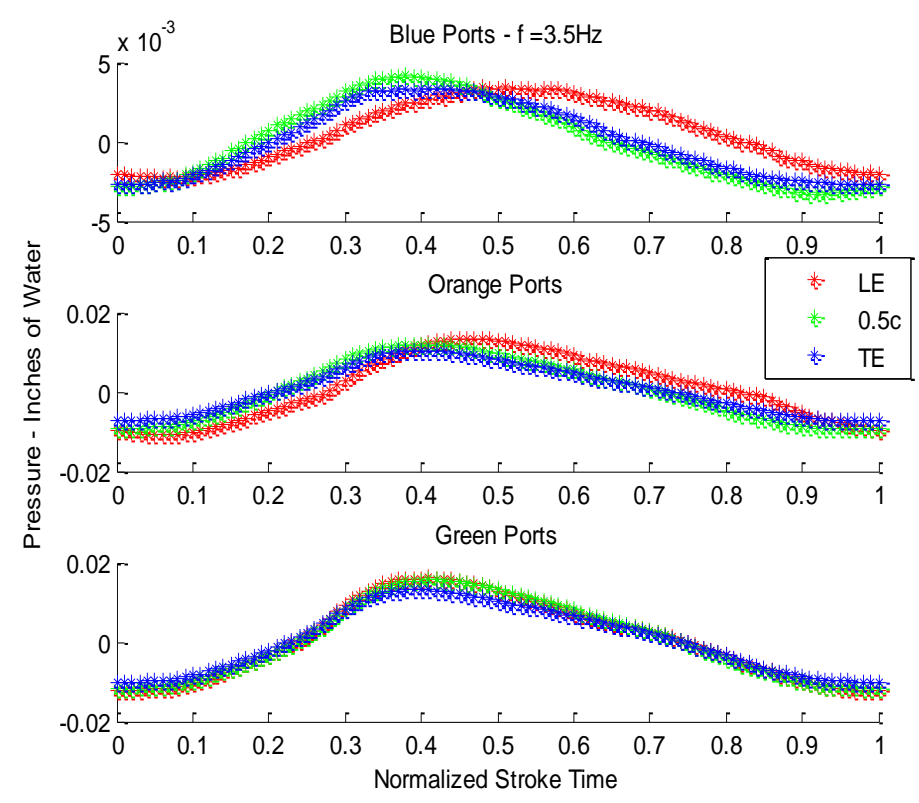

Figure 9: Example of Pressure Measurements Over Left Wing f=3.5Hz

It can be noted that the pressure distributions do not show appreciable variation between leading edge, mid-chord and trialing edge wing locations. This indicates the absence of leading edge suction. From previous sets of forward flight pressure results [36], leading edge suction is indicated by the red dots indicating data from the leading edge ports reporting larger pressure magnitudes throughout the stroke. This observation corresponds to our expectation of fully separated flow across the wing at hover and the current set of data is consistent with measurements taken during previous tests. Estimating aerodynamic loads using these pressure measurements is straightforward as the wings used are rigid and the stroke kinematics known. Since the motion is a pure flap, the orientation of the wing surfaces is also always known. As described in section III, the pressure ports give measurements of differential pressure across the surface of the wing these are easily integrated across the coarse grid and resolved based on the flap angle of the wing. A compilation of the aerodynamic force histories for the four test cases are shown below. It can be seen that the magnitude of the pressure forces increase with frequency as expected. At each frequency, a kink is observed at approximately $\mathrm{t}=0.75$. This is due to the non-symmetrical flap stoke and is an expected trend. 


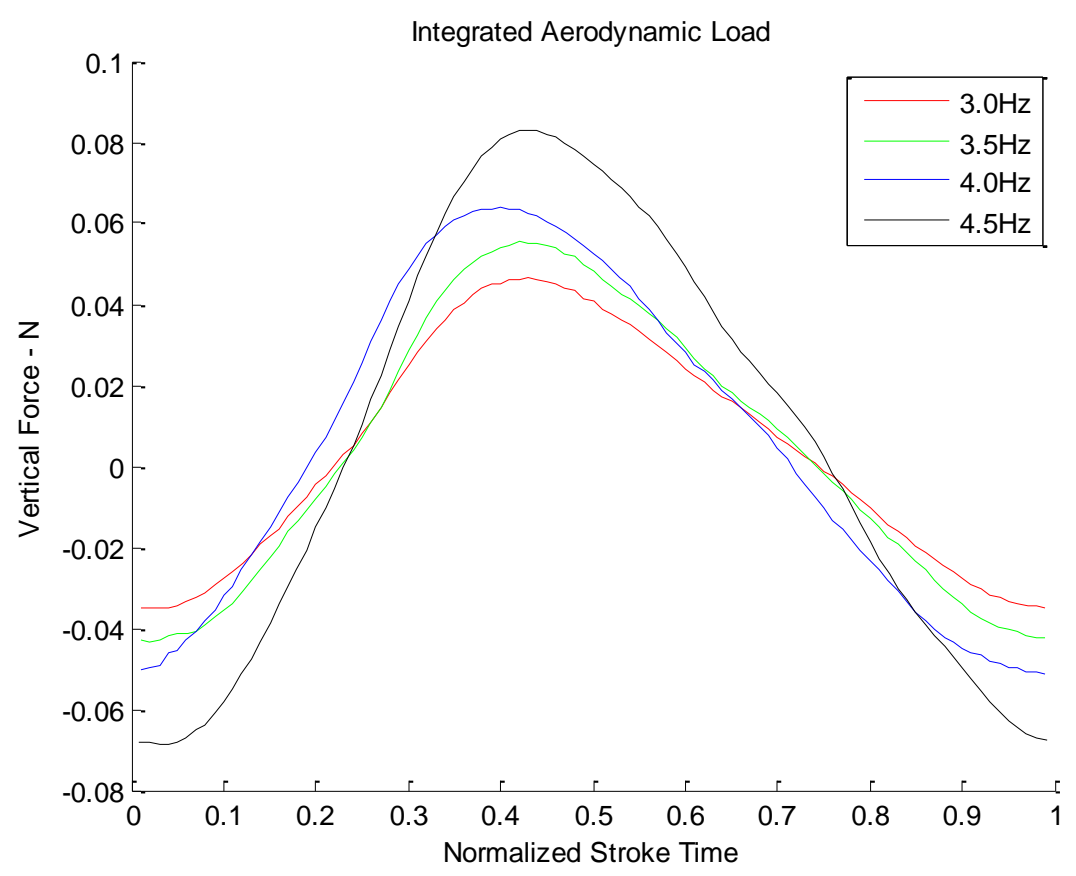

Figure 10: Pressure Measurement Based Aerodynamic Force Estimates

In order to experimentally verify the ability of the pressure based aerodynamic feedback system, measurements of the same vertical force taken using the ATI FT sensor need to be compared and checked for agreement to these pressure-based estimates. The techniques used to acquire and process the force measurement data from the vacuum chamber and wind tunnel tests are documented in the following sections.

\section{Frequency Domain Analysis of Force Torque Data}

The measurements taken by the Force Torque sensors include contributions from the structure including structural modes of the flapping wing vehicle and the test stand itself. In order to take measurements of the aerodynamic forces alone, these structural contributions that arise from the test stands structural response need to be separated from the overall signal. With force data acquired from vacuum and air, a frequency domain analysis of measurement signals was carried out to document the various periodicities present in the data. By comparing the data between multiple cases in different test environment, the causes behind the different periodicities can be identified. The results of this analysis were used to establish the post processing filter parameters for the data. Once modes are determined to be the result of the flapping motion, test stand structural response, or the aerodynamics, appropriate filter settings that exclude unwanted effects can be selected. As vertical forces are the primary measurement for this analysis, the power spectrum of the $\mathrm{Z}$ axis force component was considered in processing all data.

\section{Test Stand Structural Response - Vacuum Data only}

First, periodicities associated with the flap stands structural response were sought. To get a basic idea of what flapping related data could be expected to look like, An 'analytical version' of the test was run using the simulated kinematics from the 3-dimensional linkage solver written for the test mechanics. Since the flapping kinematics are accurately represented by a $4^{\text {th }}$ order fourier fit, 4 peaks are expected and this is shown in the spectrum below for $4.5 \mathrm{~Hz}$. It was noted from the baseline power spectrum that the contribution from the $4^{\text {th }}$ mode is relatively small compared to the first three. 


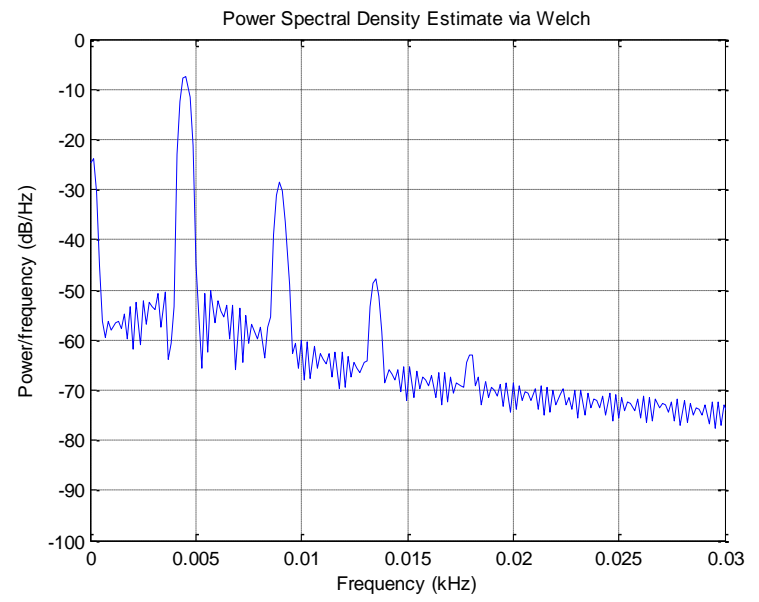

Figure 10: Sample Power Spectrum from a Simulated Test Run at $4.5 \mathrm{~Hz}$

The power spectra of data taken in a vacuum at different frequencies were then generated using the pwelch function in Matlab which uses Welch's method to compute the power spectral density of a given signal. These experimental spectra were compared to the simulated version and it was noted that the four peaks at multiples of the flapping frequency seen in the analytical case were not generally encountered in the experimental data. Shown below in figure 11 are two sample power spectra of the vertical force component in vacuum. Only the first two were apparent in all test cases. From the data, it also appeared that a set of three periodicities at higher frequencies about 8 times the flap frequencies were present. However, these did not turn out to always be at frequencies that were multiples of the main flap frequency. Since no significant aerodynamic forces were present in vacuum, only periodicities up to 4 times the flap frequency are expected due to the kinematics. As such, these periodicities at high frequencies were determined to be due to the structural response of the flap stand and needed to be filtered.
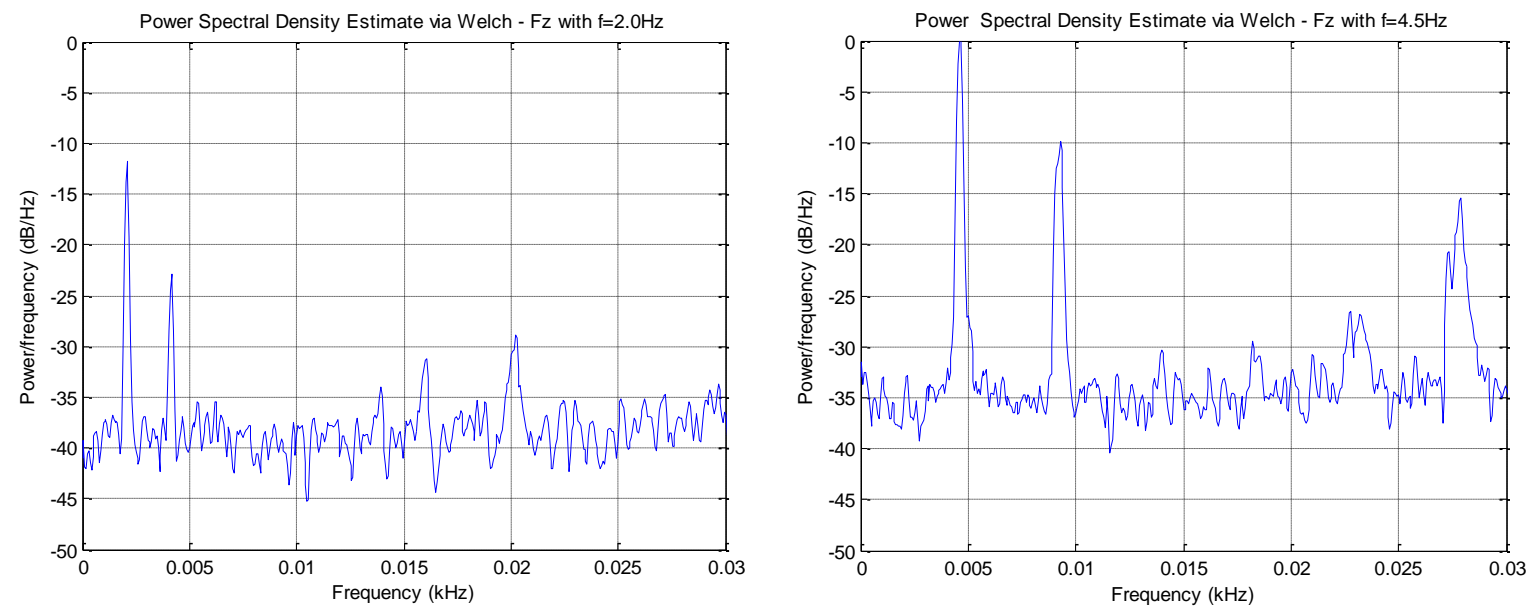

Figure 11: Sample Power Spectrum Comparison Between Flap Frequencies in Vacuum: $2.0 \mathrm{~Hz}$ (Left) and 4.5Hz (Right)

More care was taken in considering a periodicity that was noted at approximately $14 \mathrm{~Hz}$. It appeared to be the result of the flap stands response but it was not always apparent. It was possible that it was a mode of the flapping forces. $14 \mathrm{~Hz}$ and could have simply been the $4^{\text {th }}$ harmonic from the flapping stroke when flapped at $3.5 \mathrm{~Hz}$ or close to the third harmonic at $4.5 \mathrm{~Hz}$. However, from the analytical case the peak resulting from the $4^{\text {th }}$ harmonic is small compared to the third harmonic and a $3^{\text {rd }}$ flapping harmonic was never observed unless it coincided with the $14 \mathrm{~Hz}$ mode in question. When operating at $2 \mathrm{~Hz}$, the peak at $14 \mathrm{~Hz}$ was also observed even though $14 \mathrm{~Hz}$ is too high to be related to the main flapping frequency. Based on these two observations, it was thus considered likely that the mode at $14 \mathrm{~Hz}$ was due to the structural response of the test stand. The modes observed throughout a frequency sweep in vacuum are summarized in Table 5 below. Due to the non-flapping related mode at $14 \mathrm{~Hz}$, it was determined that a low pass filter with a cut off frequency that was lower than $12 \mathrm{~Hz}$ would be needed in order to remove the contribution of test-stand structural response from the inertial force torque measurements. While a filter cut off 
frequency of 4 times the flap frequency was thought to be necessary to preserve all information, the contributions of the $3^{\text {rd }}$ and $4^{\text {th }}$ harmonic did not appear to be significant in practice, suggesting that no major flap force information was contained within the raw data at frequencies 3 and 4 times the flap frequency. A filter cut off that was 2 times the flap frequency was chosen for all the vacuum chamber cases.

\begin{tabular}{|c|c|c|c|c|c|c|c|}
\hline $\begin{array}{l}\text { Flap } \\
\text { Freq }\end{array}$ & \multicolumn{7}{|l|}{$\begin{array}{l}\text { Mode } \\
\text { Freq }\end{array}$} \\
\hline $2.0 \mathrm{~Hz}$ & 2.075 & 4.15 & 13.9 & 16.05 & 20.2 & & \\
\hline $2.5 \mathrm{~Hz}$ & 2.63 & 5.19 & & & 20.32 & 25.3 & \\
\hline $3.0 \mathrm{~Hz}$ & 3.17 & 6.29 & & & 21.12 & 24.05 & \\
\hline $3.5 \mathrm{~Hz}$ & 3.48 & 6.96 & 13.92 & & & 23.25 & 26.73 \\
\hline $4.0 \mathrm{~Hz}$ & 4.08 & 8.3 & 12.39 & 16.48 & 20.81 & 24.96 & \\
\hline $4.5 \mathrm{~Hz}$ & 4.58 & 9.27 & 13.98 & 18.19 & & 23.19 & 27.83 \\
\hline
\end{tabular}

\section{Identification of Modes Caused by Aerodynamic Forces - Comparing Air and Vacuum Data}

It was anticipated that aerodynamics might contribute to periodicities at higher frequencies than the main flap frequency. It is necessary to identify these modes so filter settings that do not interfere with aerodynamic data can be chosen around them. In order to identify modes due to aerodynamics, power spectrums for air and vacuum data taken at the same flapping frequency are compared and additional modes in the air cases are sought. However, it was found when comparing the power spectrums of tests run in vacuum and in air that no additional peaks were discernible when aerodynamic forces were present. It was determined that no additional 'aerodynamic modes' were apparent and that filter settings for the vacuum and air data could be the same. For the data presented in this paper, a low pass filter with a cut off frequency of 2 times the main flap frequency was used for both in-air and vacuum cases. A sample comparison at $3.0 \mathrm{~Hz}$ is shown below.
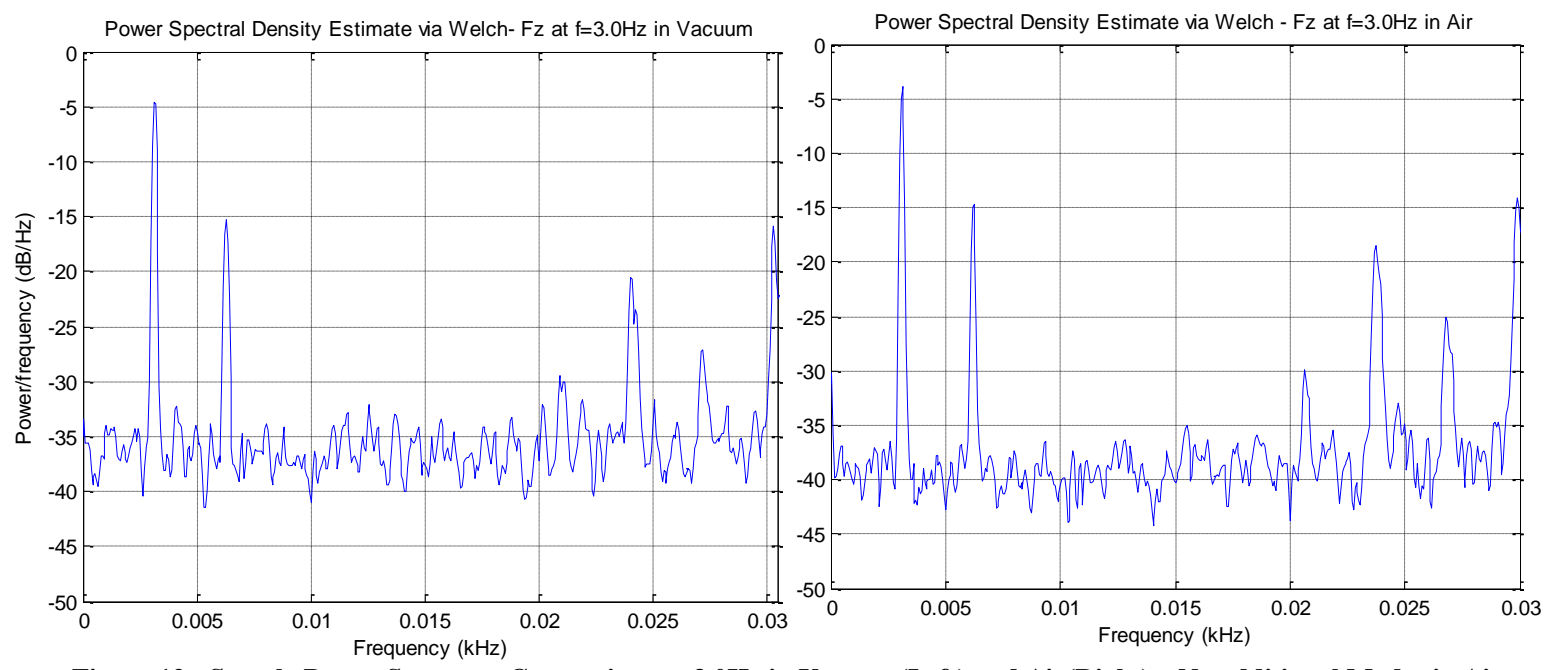

Figure 12: Sample Power Spectrum Comparison at 3.0Hz in Vacuum(Left) and Air(Right) - No additional Modes in Air

\section{Force Data Processing and Inertial Load Subtraction Methodology}

In order to isolate the aerodynamic loads from the inertial loads, the results from two different force measurement experiments needed to be combined correctly. The approach taken was to process the data from each test case individually and obtain a characteristic stroke for the vacuum chamber and wind tunnel data. The characteristic stroke from the vacuum chamber test is then subtracted from the characteristic stroke of the wind tunnel test case for a characteristic aerodynamic load at a given flap frequency. 


\section{Ensemble Averaging Process for Force Measurements}

For each test case, the mechanism was flapped for an interval of 30-40seconds to allow multiple flap cycles to be captured. The force measurements are then filtered using a Butterworth filter implemented in Matlab with a low pass cut-off that is 2 times the flap frequency based on the analysis described in Section VIII. Data from the magnetic encoder was used to identify individual flap strokes and split the filtered force data set into multiple single-flap measurements. A time scale is then used to establish along a normalized period and the data from each trial is overlaid on this temporal grid. If test data was not available at a particular point in time along the normalized period, a linear interpolation between neighboring points was used in its place. The result of this is a normalized flap period with each point in time containing a number of measurements from the number of flap cycles captured. These sample populations are then used to determine the statistics at each point in the normalized period assuming a normal distribution. A sample result is shown below showing the differences in error as more flaps are used in the data. The blue dots denote the $95 \% \mathrm{CI}$ around the ensemble averaged values. The plots along the bottom show the diminishing width of the $95 \%$ confidence interval as the number of flaps considered is increased from 4 to 138 . When generating the following plots, the normalized time scale is split into 100 intervals for clarity.
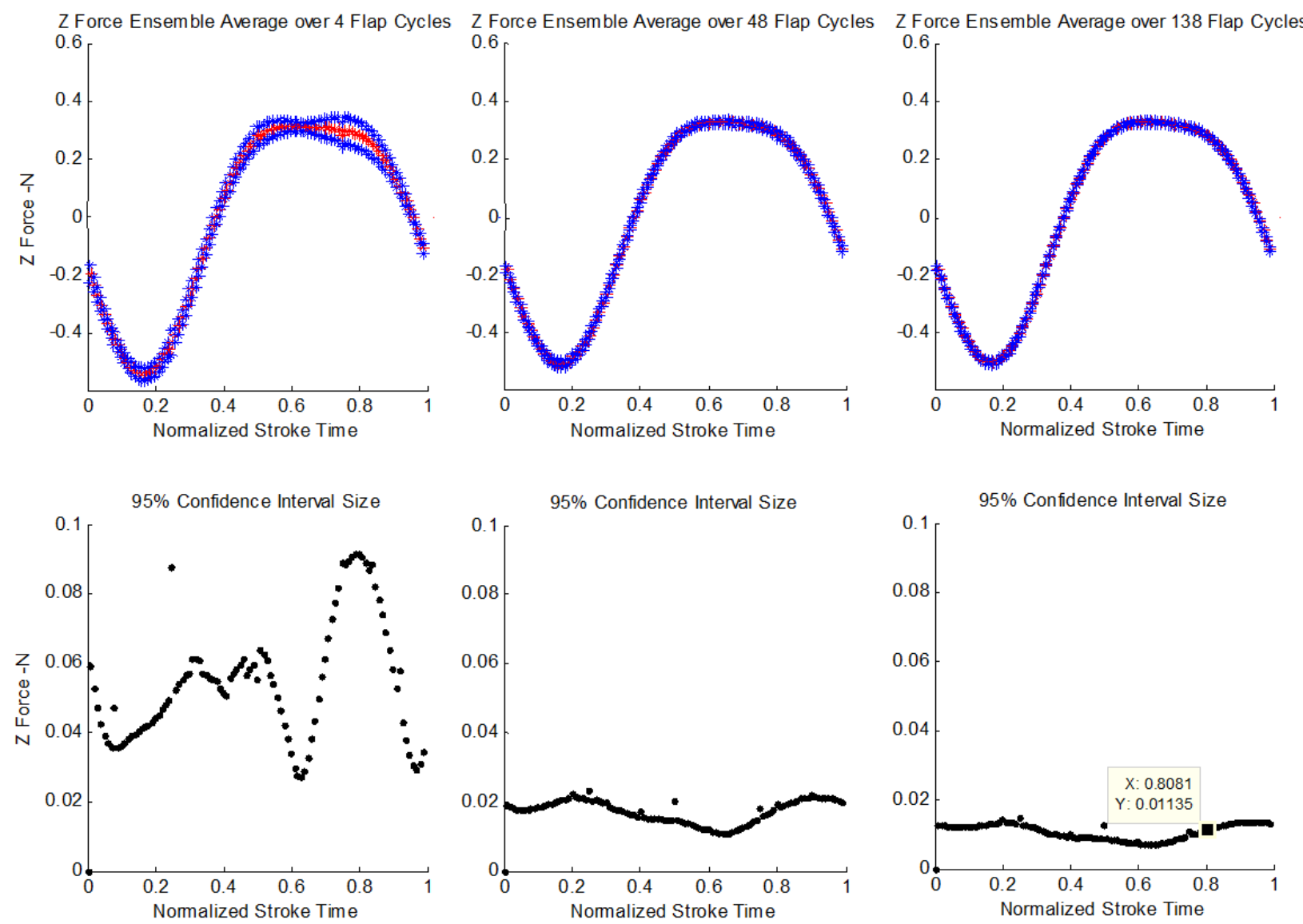

Figure 13: Ensemble Averaging Process for Force Data and 95\% CI - 3.5Hz in Vacuum

\section{Verification of Single-Stroke Aerodynamic Force History using Cycle Averaged Forces}

In combining the data from three different tests, a number of challenges were encountered in obtaining the best measurement of aerodynamic forces. Most significantly, small errors in phase synchronization between the cases when subtracting loads between the vacuum loads from wind tunnel loads could lead to large errors in aero force estimates. For example, a 3\% phase error could lead to complete reversal of aerodynamic force histories due to the nature of the load subtraction process. The plots in Figure 14 depict the effect of a small change in encoder timing on the resulting aerodynamic estimates. 

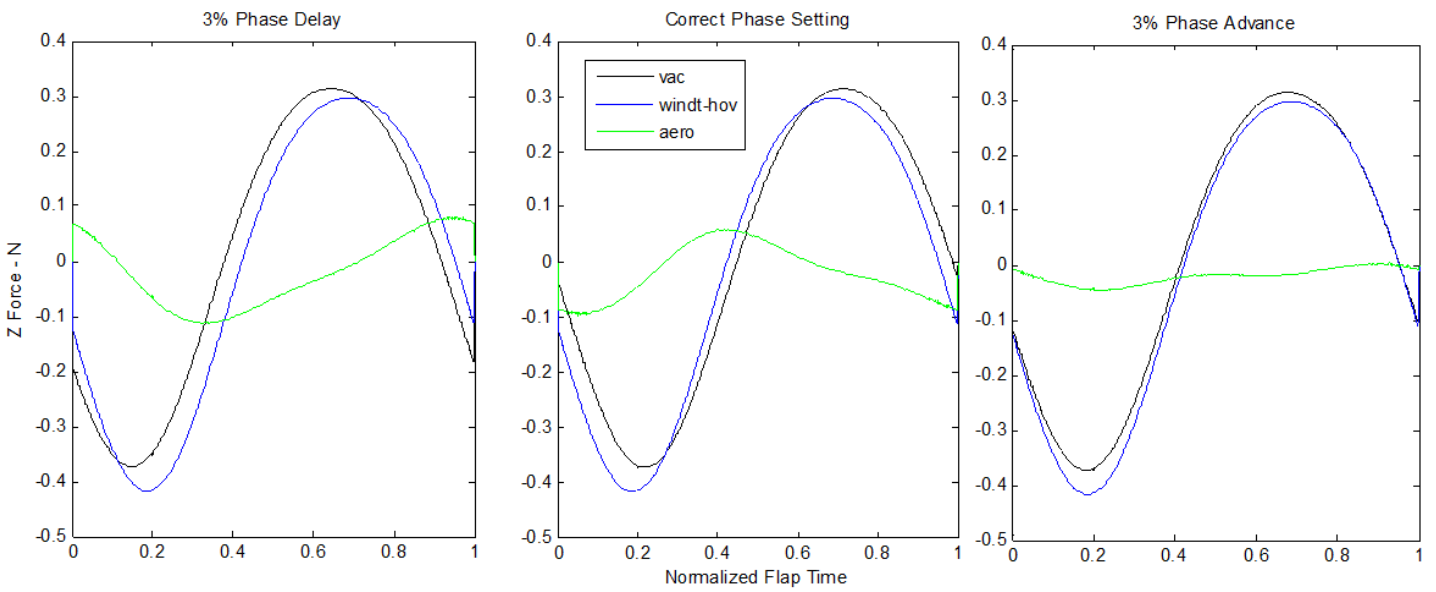

Figure 14: Sensitivity of Measurement to Encoder Phase Offset $-\mathbf{3 . 0 H z}$

In order to provide a secondary means of verifying the subtracted aerodynamic force data is consistent with the forces measured in air and in vacuum, cycle averaged forces were considered. Since the cycle average force measurements of the raw data taken over a long period are less dependent on filter settings and potential encoder offsets, they offer a more reliable alternative measurement with which to evaluate the single-stroke results and to ensure that the force measurements are consistent with themselves.

The unfiltered data was first used to compute the average vertical force in either test case. The average force in a vacuum throughout the total number of flaps was subtracted from the average force in air to provide an estimate of the cycle averaged aerodynamic force for that flapping frequency. This was then used to verify that the subtracted single-cycle aerodynamic loads resulted in the same cycle averaged aerodynamic loads from the subtracted aerodynamic load histories. This technique verified that the flap cases run at the four frequencies provided an accurate estimate of single-cycle aerodynamic forces that were within 5\% of the cycle averaged measurements. The resulting data from this comparison is compiled in Table 6.

Table 6: Cycle Averaged and Single-Stroke Aerodynamic Force Errors

\begin{tabular}{|c|c|c|c|c|c|c|}
\hline \multicolumn{7}{|c|}{ Fz Averages } \\
\hline Freq & Windtunnel & Vac & Target & Offset & Single Flap Final & Error \\
& $(\mathrm{N})$ & $(\mathrm{N})$ & air-vac (N) & $(\mathrm{ms})$ & $(\mathrm{N})$ & \\
$3.0 \mathrm{~Hz}$ & $1.411 \mathrm{E}-02$ & $5.795 \mathrm{E}-03$ & $1.991 \mathrm{E}-02$ & vac-0 & $2.07 \mathrm{E}-02$ & $\sim 4 \%$ \\
$3.5 \mathrm{~Hz}$ & $-3.153 \mathrm{E}-04$ & $-6.642 \mathrm{E}-03$ & $6.327 \mathrm{E}-03$ & vac-0 & $6.47 \mathrm{E}-03$ & $\sim 2 \%$ \\
$4.0 \mathrm{~Hz}$ & $2.660 \mathrm{E}-04$ & $-1.553 \mathrm{E}-02$ & $1.580 \mathrm{E}-02$ & vac-0 & $1.52 \mathrm{E}-02$ & $\sim 4 \%$ \\
$4.5 \mathrm{~Hz}$ & $-2.112 \mathrm{E}-03$ & $-8.646 \mathrm{E}-03$ & $6.534 \mathrm{E}-03$ & vac-0 & $6.46 \mathrm{E}-03$ & $\sim 1 \%$ \\
\hline
\end{tabular}

\section{Confidence Intervals on Subtracted Aerodynamic Loads}

While an error analysis is important in any experiment, the challenging nature of obtaining a measurement of the aerodynamic force from two sets of data from two completely different environments made an investigation of the errors more critical. The test stand and pressure measurement system are intended for forward flight conditions such as those presented in previous work [35,36]. The aerodynamic loads encountered at hover are much smaller and approach the resolution of the ATI Nano 17 sensor. Further, the data from two different test runs are combined so the error of both individual test runs must also be combined. If the interval of uncertainty around the measured aerodynamic force estimates were too big relative to the data itself, the usefulness of the test setup would need to be reassessed. The statistics of each set of FT measurement results are computed at each point in the normalized characteristic stroke by considering the total number of data points available and assuming a standard distribution. In this manner, the standard deviation is computed for each point in the characteristic average stroke from wind tunnel test and vacuum chamber tests. When the inertial loads are subtracted, the standard deviations are added. The combined standard deviation can then be used to provide an estimate of the confidence interval around the final data 
point. The following plots present the standard deviation intervals encountered from different parts of a normalized flap stroke.
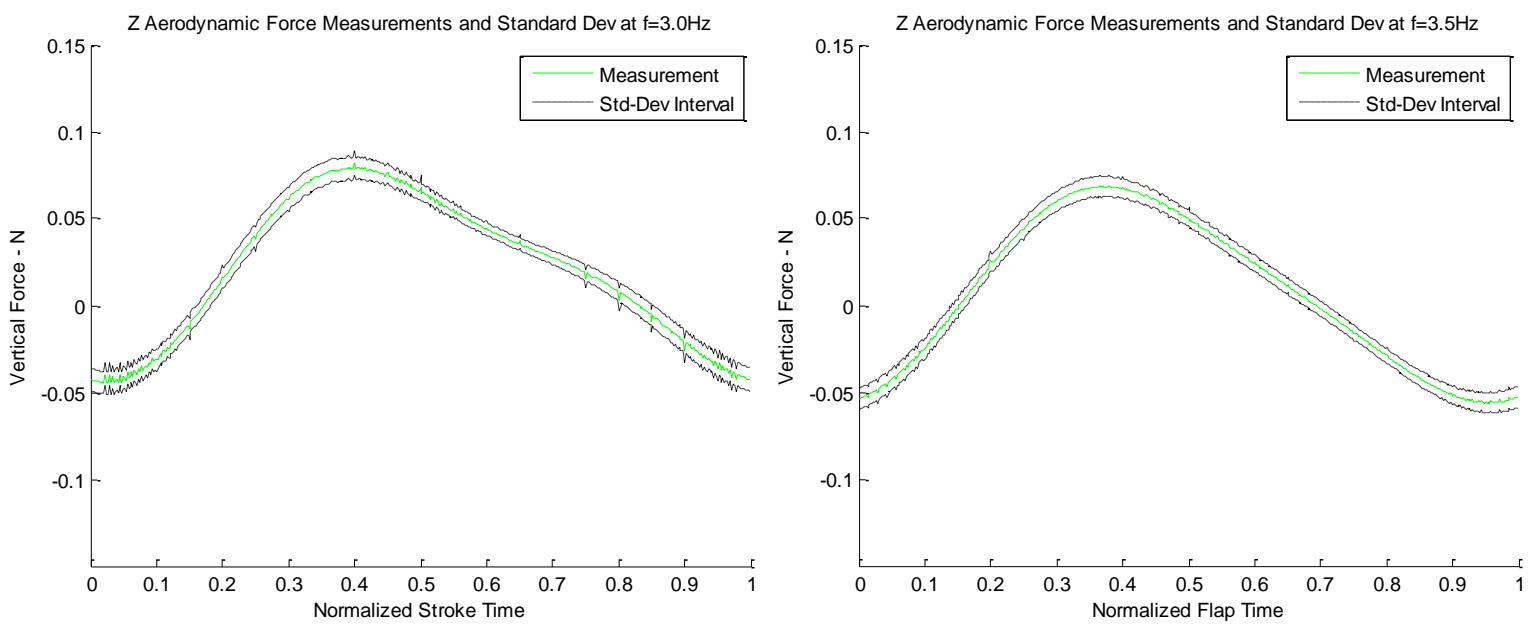

Figure 15: Standard Deviation Intervals on Subtracted Aerodynamic Load Measurements $-\mathrm{f}=3.0$ and 3.5Hz

The plots above present data from the two slower cases which have the two biggest relative standard deviation intervals. This is due to smaller overall measurements and sample sizes during test runs of fixed duration. Even so, the average trends and magnitudes are not obscured when surrounded by the standard deviation interval. This suggests that the hover data yielded reasonable error statistics despite previous concerns about sensor performance at the lower aerodynamic forces encountered at hover. From the standard deviation interval shown above, it is expected that the hover data will be useful in developing data processing and comparison methods for future forward flight tests.

\section{Comparison of Pressure Based Estimates and Force Sensor Aerodynamic Force Measurements}

The basic requirement of an aerodynamic feedback system is the ability to provide a real time estimate of the aerodynamic loads. In order to assess the value of the pressure based aerodynamic sensing system, single stroke $\mathrm{Z}$ force history measured using the FT sensor and estimated using the pressure measurements need to be compared. The data presented in the following comparisons are generated with the wing phase shown in figure 16 below. The normalized stroke begins with the wings crossing their midpoint flap angle while on their upstroke. At about $\mathrm{t}=0.25$, the wings reach their top most position and begin their down stroke. Due to asymmetry in the stroke, the wing decelerates more slowly at the bottom of the down stroke and spends more time in its bottom position between $\mathrm{t}=0.7$ and $\mathrm{t}=0.8$ before finally beginning the upstroke again.

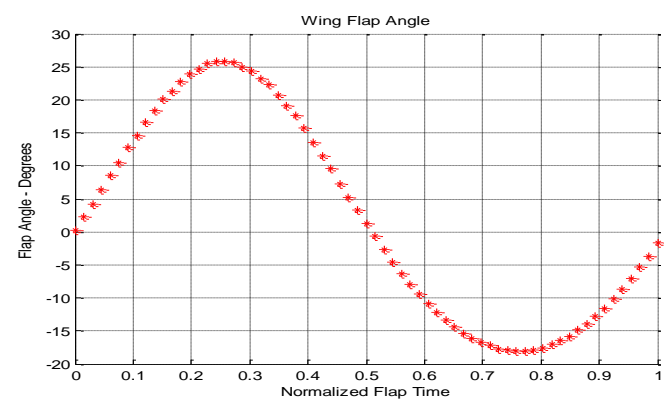

Figure 16: Wing Kinematics - Positive Flap Angles Denote Wings Above Level

\section{Direct Force Comparison}

The first step in the process was to directly compare the best-guess results of force torque measurements to the filtered pressure measurements. These are overlaid in the following plots. From the comparison, it can be seen that 
the pressure based estimates exhibit similar trends and track the measured vertical forces. At $t=0.7$ a change in slope of aerodynamic loads are encountered in both sets of data. At the same time, distinct differences are apparent. The magnitudes estimated by the pressure based aerodynamic data system are smaller compared to those reported by the force measurements. There is an approximate $10-15 \%$ phase lag in the pressure estimates for the test cases. This suggests that a certain amount of information is indeed being lost by the pressure based estimates as only aerodynamic forces can explain the difference between the cycle-averaged forces in air and in vacuum. Since the pressure instrumentation appears to correctly track the forces being generated, these results suggest that the pressure measurement system has the ability to provide a basic estimate of aerodynamic forces and is feasible but requires further investigation.
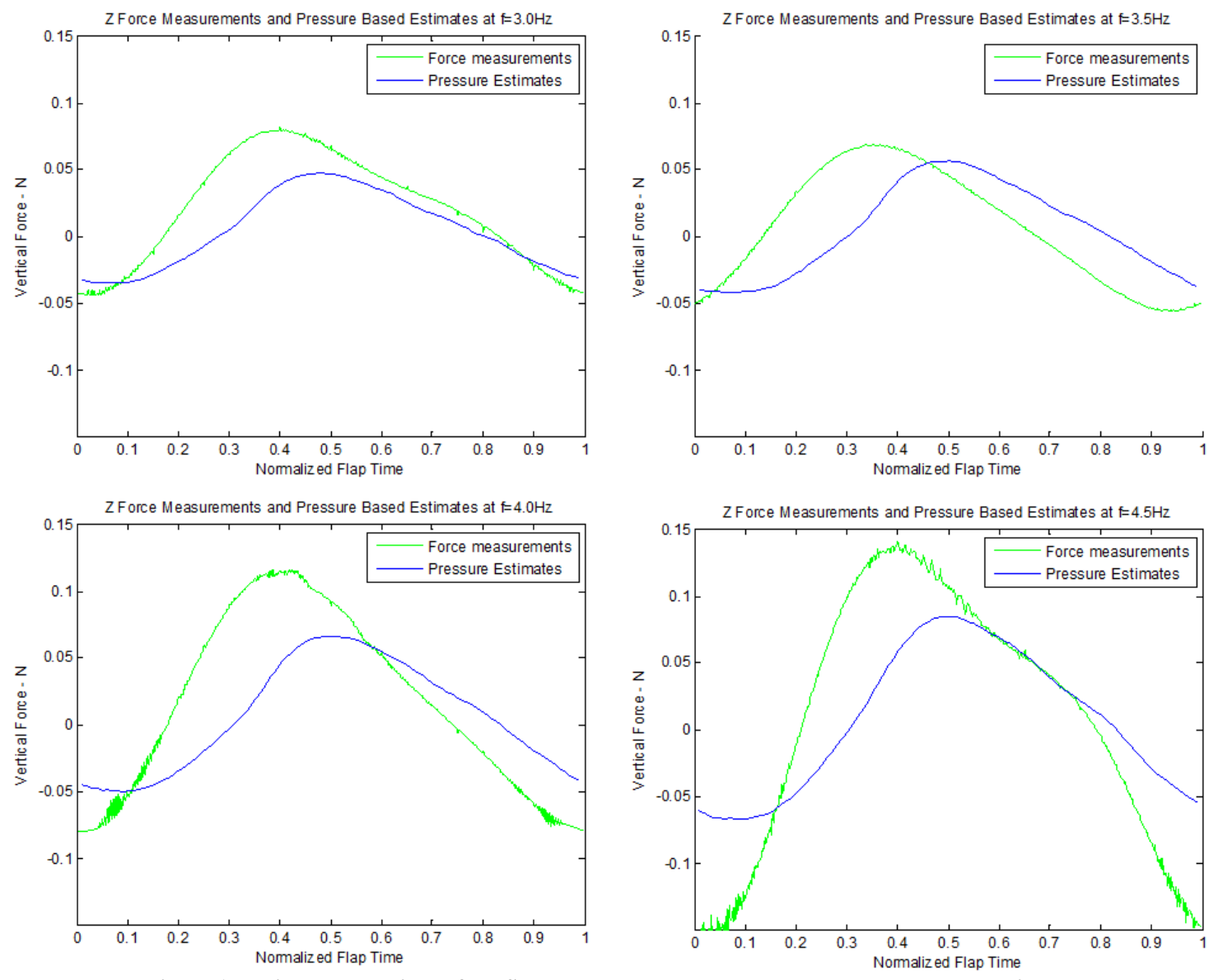

Figure 17: Direct comparison of FT Sensor Measurements and Pressure Based Force Estimates

As the calibration of the pressure and force instrumentation has been checked for agreement using a steady fixed NACA wing test case for reference, a calibration error is not expected to be the cause of the observed discrepancies. The most probable cause is thought to be the length of the pressure lines used. Due to the large volume of air in them and the relatively small pressure port size on the wing, the pressure lines behave like a physical low-pass filter. It was thought that this low-pass filter effect in the pressure lines would explain the differences in trend and the smaller predicted force magnitudes.

\section{Second Comparisons with Shorter Pressure Line Lengths}

To explore this possibility further, a secondary set of hover data was taken with the pressure lines shortened from $1.3 \mathrm{~m}$ to $0.3 \mathrm{~m}$ as a pre-cursor to a short set of test runs with different pressure line lengths. In order to facilitate these new pressure line lengths, the pressure sensor tray had to be temporarily moved from its designed position behind the flap stand and attached on the back of the main arm. It was hypothesized that if the discrepancies were due to this physical mechanism, the dramatic reduction in pressure line length should allow the aerodynamic predictions to 
more closely match the modified force measurements. The results of this comparison are shown in figure 18 . When a short pressure line length is used a noticeable improvement in phase error is observed. This is accompanied by larger magnitudes predicted by the pressure based measurement system and a better overall match throughout the test cases.
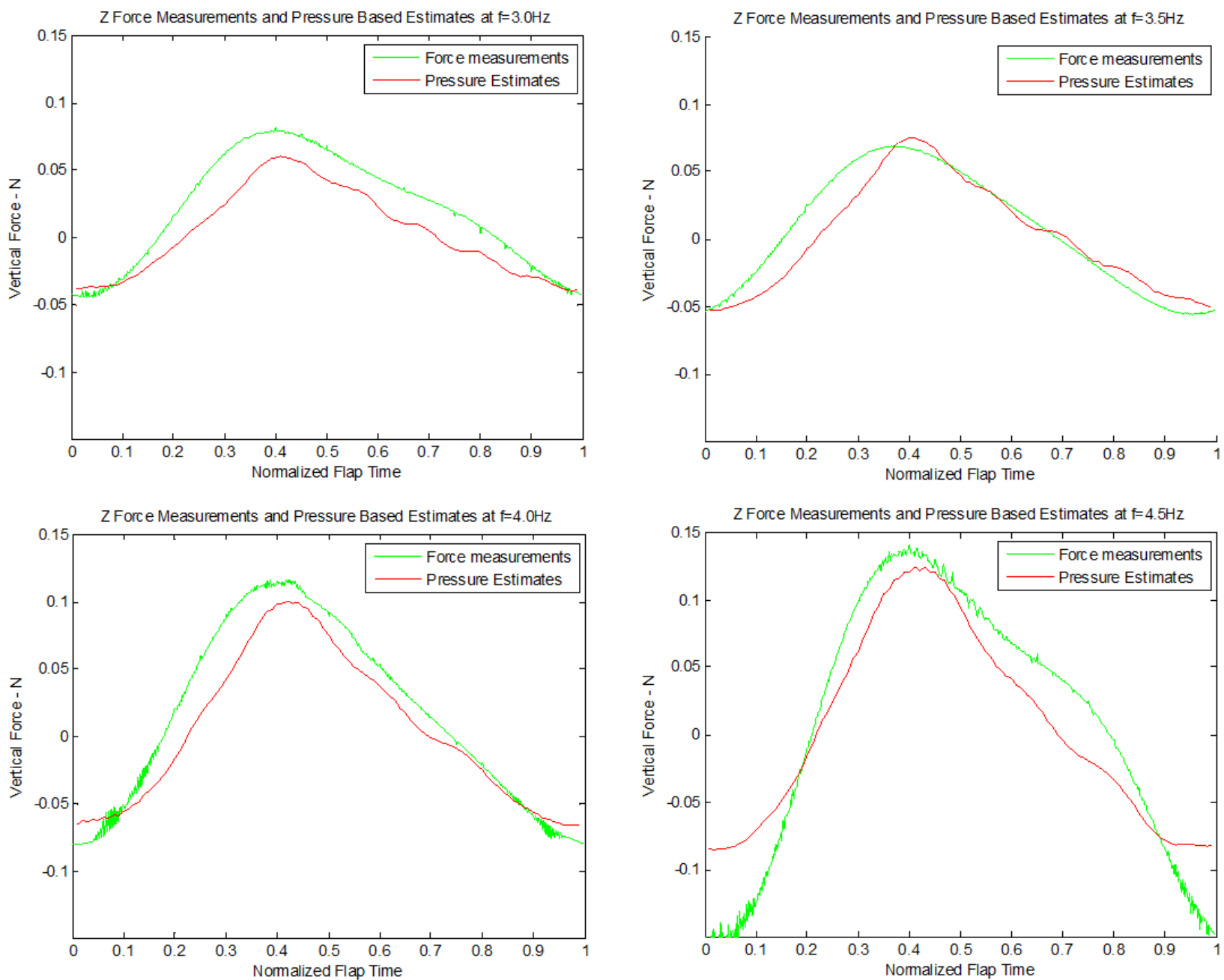

Figure 18: Second Comparison with Lower LP Cut-off Frequencies on Force Measurements

The second comparison supports the hypothesis that the additional low-pass filter effects explained some of the main discrepancies observed when using the original flap stand setup. This result indicates that the current pressure measurement system is capable of estimating aerodynamic loads in a general manner and the approach is feasible. While main trends in both sets of data are comparable, some discrepancies remain. Peak magnitudes for $3.0 \mathrm{~Hz}$, $3.5 \mathrm{~Hz}$ and $4.0 \mathrm{~Hz}$ are a better match but large differences throughout the cycle still exist. At the highest frequency of $4.5 \mathrm{~Hz}$, an unexpectedly large negative peak in the measured force data is observed that is not reflected in the pressure measurements. It was noticed that the pressure measurements were consistent in phase between the different frequencies, while the force measurement history for the $3.5 \mathrm{~Hz}$ case appeared to have a phase advance when compared to force measurements from other test frequencies. The encoder settings for the three cases were verified against the wing position data for each test condition at $3.5 \mathrm{~Hz}$. One possible cause is a larger spread in flap stroke period during the force measurement tests resulting in errors when computing the average stroke. This suggests that the force measurements need further investigation as well.

A complementary set of computational cases are also being used in ongoing work to serve as another basis for evaluating the aerodynamic force measurements and pressure based estimates using the CFD framework established and used by Kang et al in [17] and the approximate method described by Gogulapati et al in [16]. 


\section{Conclusions}

This paper has presented an experimental investigation of the feasibility of implementing a pressure based aerodynamic data system on a flapping wing vehicle platform for the purpose of making real-time estimates of the aerodynamic forces generated by the wing. Two different measurements of the vertical aerodynamic loads generated by a rigid flapping wing in hover have been acquired at $\mathrm{Re}=3500$ to $\mathrm{Re} 5500$ using a force torque sensor and a set of embedded pressure instrumentation across the wing surface. Our findings include the following:

- Use of a rigid wing with repeatable kinematics for experiments conducted in both air and vacuum has enabled us to separate inertial loads from aerodynamics forces and torques generated by flapping.

- Experimental comparisons suggest the pressure based aerodynamic data system is able to estimate aerodynamic forces over a rigid flapping wing but with some limitations in performance.

- Initial comparisons with the original long pressure line configuration showed that the pressure based estimates were estimating smaller loads than those measured and involved a noticeable 10-15\% phase lag. It is hypothesized that the discrepancies are due to the frequency response of the pressure measurement system is caused by the large volumes between the wing ports and the pressure transducers.

- Secondary tests with a shortened pressure line configuration show that comparable overall trends, peak magnitudes and phase are captured by both sets of measurements. However, smaller magnitudes are reported by the pressure measurement system throughout the cycle.

- The pressure based estimates exhibited expected trends in peak magnitude while unexpected trends in the force measurements are noticeable in the $4.5 \mathrm{~Hz}$ case. This may be due to the relative sensitivity of the force measurements to vibrations in the stand, and suggest that pressure based estimates might be useful in providing complimentary results when further evaluating the force measurement setup. 


\section{References}

1 W.Shyy, Y. Lian, J. Tang, D. Viieru, and H. Liu, Aerodynamics of Low Reynolds Number Flyers, Cambridge University Press, 2007.

2 R. Wood, "The First Takeoff of a Biologically Inspired Robotic Insect" IEEE Transactions On Robotics, Vol.24, No.2, April 2008.

3 D.Mueller, H.A. Bruck, S.K. Gupta, "Measurement of Thrust and Lift Forces Associated With Drag of Compliant Flapping Wing for Micro Air Vehicles Using a New Test Stand Design", J. Experimental Mechanics. vol. 50, pp. $725-735,2010$

4 K. De Clercq, R. de Kat, B. Remes, B. van Oudheusden, and H. Bijl, "Flow Visualization and Force Measurements on a Hovering Flapping-Wing MAV 'DelFly II'," Proc. 39th AIAA Fluid Dynamics Conference, 22-25 June 2009, San Antonio, Texas. (AIAA-2009-4035)

5 D. Dawson, “ Repeatable Manufacture of Wings for Flapping Wing Micro Air Vehicles Using Microelectromechanical Systems (MEMS) Fabrication Techniques" Masters thesis, Air Force Institute of Technology, March 2011

6 D. Li, S. Guo, N. Matteo, D. Yang, "Design, Experiment and Aerodynamic Calculation of a Flapping Wing Rotor Micro Aerial Vehicle", Proc. 52 ${ }^{\text {nd }}$ AIAA Structures, Structural Dynamics and Materials Conference, 4-7 April 2011, Denver Colorado (AIAA 2011-1988)

7 J. Grauer and J. Hubbard Jr. "Development of a Sensor Suite for a Flapping-Wing UAV Platform," Proc. $46^{\text {th }}$ AIAA Aerospace Sciences Meeting, January 2008. (AIAA 2008-224)

8 Z. Jackowski directed by R. Tedrake, Design and Construction of an Autonomous Ornithopter, MS Thesis, Massachusetts Institute of Technology, June 2009.

9 J. Lee, D. Lee and J. Han, "Ornithopter Attitude Estimation: Ground Test" World Automation Congress, pp. 1-6 19-23 September 2010.

10 R. Kranashita, D. Silin, S. Shkarayev, "Flight Dynamics of Flapping-Wing Air Vehicle" Proc, 49 ${ }^{\text {th }}$ AIAA Aerospace Sciences Meeting, January 2009 Reno, NV. (AIAA 2008-6698)

11 J. Han, J. Lee, and D. Kim, "Bio-inspired Flapping UAV Design: A University Perspective," SPIE, Vol. 7295, 2009.

12 Festo (2011, Mar,) Festo. [Online]. http://www.festo.com/cms/en_corp/11369.htm

13 S.Ansari, R.Zbibowski, and K.Knowles, "Non-linear Unsteady Aerodynamic Model for Insect-like Flapping wings in the Hover. Part 1" Proceedings of the Institution of Mechanical Engineers, Part G: Journal of Aerospace Engineering, vol. 220, no. 2, pp. 61-83, 2006

14 D.J.Willis, J.Peraire and J.K.White, "FastAero - a Precorrected FFT - Fast Multipole Tree Steady and Unsteady Potential Flow Solver," presented at the SMA Symposium, Singapore 2005.

15 H. Aono, S.K Chimakurthi, P. Wu, E.Sällström B.K. Stanford, C.E.S. Cesnik, P. Ifju, L, Ukeiley, and W. Shyy, " A Computational and Experimental Study of Flexible Flapping Wing Aerodynamics," Proc. 48th AIAA Aerospace Sciences Meeting Including the New Horizons Forum and Aerospace Exposition, Orlando, Florida, (AIAA-2010-554).

16 A. Gogulapati and P. Friedmann, "Approximate Aerodynamic and Aeroelastic Modeling of Flapping Wings in Hover and Forward Flight," Proc. 52nd AIAA/ASME/ASCE/AHS/ASC Structures, Structural Dynamics and Materials Conference 19th AIAA/ASME/AHS Adaptive Structures Conference, Denver, CO, 2011. (AIAA-20112008)

17 C. Kang, H. Aono, C. Cesnik and W. Shyy, "Effects of Flexibility on the Aerodynamic Performance of Flapping Wings,” J. Fluid Mech. (2011), vol. 689, pp.32-74, 2011

18 S. Walker, A. Thomas, G. Taylow, "Deformable Wing Kinematics in the Desert Locust: How and Why do Camber, Twist and Topography Vary Through the Stroke?" J R Soc Interface 2008; 6 (38): 735-747

19 Wang H, Zeng L, Liu H, Yin C. Measuring wing kinematics, flight trajectory and body attitude during forward flight and turning maneuvers in dragonflies. J Exp Biol 2003; 206: 745-757.

20 D. Read, J. Hover, M. Triantafyllou, "Forces on Oscillation Foils for Propulsion and Maneuvering," J. Fluid Struct., vol. 17, pp. 163 -- 93, 2003. 
21 Y. Baik, J. Rausch, L. Bernal, and M. Ol, "Experimental Investigation of Pitching and lunging Airfoils at

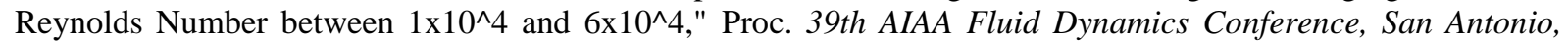
Texas, June 22-25, 2009, 2009, pp. AIAA-2009-4030.

22 K.Massey, A. Flick, and G. Jadhav, "Force Measurements and Flow Visualization for a Flexible Flapping Wing Mechanism" Proc. 47 ${ }^{\text {th }}$ AIAA Aerospace Sciences Meeting,5-8 January 2009, Orlando, Florida. (AIAA 2009877)

23 B. Singh, I. Chopra, M. Ramasamy and J. Leishman, "Insect-Based Flapping Wings for Micro Hovering Air Vehicles: Experimental Investigation," AHS International Specialists' Meeting on Unmanned Rotorcraft: Design, Control and Testing, January 2005.Phoenix, AZ.

24 P. Wu, "Deformation and Performance Measurements of MAV Flapping Wings," Proc. SEM Annual Conference June 1-4, 2009 Albuquerque New Mexico

25 D. Yeo, J. Henderson, and E Atkins, "An Aerodynamic Data System for Small Hovering Fixed-Wing UAS," Proc. AIAA Guidance, Navigation, and Control Conference, 10-13 August 2009, Chicago Illinois. (AIAA 20095756)

26 Air Force Office of Scientific Research (AFOSR) Multidisciplinary University Research Initiative (MURI) AFOSR Grant FA9550-05-1-0411, [online] http://www.avocet.gatech.edu

27 X.Deng, L. Schenato, W. Wu, and S. Sastry, "Flapping Flight for Biomimetic Robot Insects: Part 1: System Modelling," IEEE Transactions on Robotics and Automation, Vol. 22, No.4, Aug. 2006, pp.776-788.

28 X.Deng, L. Schenato, W. Wu, and S. Sastry, "Flapping Flight for Biomimetic Robot Insects: Part 2: Flight Control Design," IEEE Transactions on Robotics and Automation, Vol. 22, No.4, Aug. 2006, pp.789-803.

29 M. Bolender, "Rigid Multi-Body Equations-of-Motion for Flapping wing MAVs using Kane's Equations," Proc, AIAA Guidance Navigation and Control Conference, Chicago, IL 2009. (AIAA 2009-6158)

30 D. Doman, D. Oppenheimer, D. Sigthorrson, "Dynamics and Control of a Minimally Actuated Biomimetic Vehicle, Part1: Aerodynamic Model," Proc. AIAA Guidance Navigation and Control Conference, Chicago, IL 2009. (AIAA 2009-6160)

31 D. Doman, D. Oppenheimer, D. Sigthorrson, "Dynamics and Control of a Biomimetic Vehicle Using Biased Wingbeat Forcing Functions" Journal of Guidance, Control, and Dynamics vol.34 no.1 (204-217) 2010

32 A. Hilairw and F. Carta, " Analysis of unswept and swept wing chordwise pressure data from an oscillating NACA 0012 airfoil experiment. NASA CR-3567, 1983

33 M. Green and A. Smits, "Effects of Three-Dimensionality on Thrust Production by a Pitching Panel", J. Fluid Mech. Vol.615 pp211-220, 2008

34 H.Takahashi, Y.Aoyama, K.Ohsawa, H. Tanaka, E. Iwase, K. Matsumoto, I.Shimoyama, "Differential pressure measurement using a free flying insect-like ornithopter with a MEMs sensor" Bioinspiration and Biomimetics, vol. 5 no. 3, 036005, 2010.

35 D. Yeo, E. Atkins, W. Shyy, “Aerodynamic Sensing as Feedback for Ornithopter Flight Control," Proc. 49 $9^{\text {th }}$ AIAA Aerospace Sciences Meeting, January 2011, Orlando, Florida. (AIAA 2011-552)

36 D. Yeo, E. Atkins, and W. Shyy, "Experimental and Analytical Pressure Characterization of a Rigid Flapping Wing for Ornithopter Development," Proc. AIAA Atmospheric Flight Mechanics Conference, 8-11 August 2011, Portland Oregon.(AIAA 2011-6518) 
